\title{
Hamilton's principle and normal mode coupling in an aspherical planet with a fluid core
}

\author{
David Al-Attar, ${ }^{1}$ Ophelia Crawford, ${ }^{1}$ Andrew P. Valentine ${ }^{2}$ and Jeannot Trampert ${ }^{3}$ \\ ${ }^{1}$ Bullard Laboratories, University of Cambridge, Madingley Road, Cambridge CB3 OEZ, UK. E-mail: da380@cam.ac.uk \\ ${ }^{2}$ Research School of Earth Sciences, The Australian National University, Acton, ACT 2601, Australia \\ ${ }^{3}$ Department of Earth Sciences, Universiteit Utrecht, P.O. Box 80021, 3508 TA Utrecht, The Netherlands
}

Accepted 2018 April 8. Received 2018 April 2; in original form 2018 January 26

\begin{abstract}
SUMMAR Y
We apply Hamilton's principle to obtain the exact equations of motion for an elastic planet that is rotating, self-gravitating and comprises both fluid and solid regions. This variational problem is complicated by the occurrence of tangential slip at fluid-solid boundaries, but we show how this can be accommodated both directly and using the method of Lagrange multipliers. A novelty of our approach is that the planet's motion is described relative to an arbitrary reference configuration, with this generality offering advantages for numerical calculations. In particular, aspherical topography on the free surface or internal boundaries of the planet's equilibrium configuration can be converted exactly into effective volumetric heterogeneities within a geometrically spherical reference body by applying a suitable particle relabelling transformation. The theory is then specialized to consider the linearized motion of a planet about a steadily rotating equilibrium configuration, with these results having applications to normal mode coupling calculations used within studies of long-period seismology, tidal deformation and related fields. In particular, we explain how our new theory will, for the first time, allow aspherical boundary topography to be incorporated exactly within such coupling calculations.
\end{abstract}

Key words: Tides and planetary waves; Surface waves and free oscillations; Theoretical seismology.

\section{INTRODUCTION}

This paper presents a new formulation for the equations of motion of a self-gravitating, rotating and elastic planet as needed in studies of longperiod seismology, tidal deformation and other related problems. It builds on the work of Al-Attar \& Crawford (2016) (from here on AC16) where we introduced particle relabelling transformations, and demonstrated their potential application within computational seismology through some simple numerical examples. The results of AC16 are, however, limited to elastic bodies that are either entirely solid or entirely fluid, and so cannot be applied to planets such as the Earth possessing a fluid outer core or surface oceans. The aim of this paper is to extend the theoretical results of AC16 to fluid-solid bodies. This work has been primarily motivated by a desire to incorporate, for the first time, aspherical boundary topography into normal mode calculations in an exact manner, and it will be useful to review the background to this problem below. Potential applications of this work, however, extend beyond long-period seismology, with our approach likely being useful in other geophysical problems in which boundary topography needs to be considered. Here we note, in particular, that AC16 showed analogous particle relabelling transformations that can be applied within viscoelastic bodies. In the second part of this introduction, we provide further background on particle relabelling transformations of a more technical nature, concentrating on issues associated with fluid-solid boundaries that form the main focus of this work.

\subsection{Normal mode coupling calculations}

Within a spherically symmetric earth model, it is possible to calculate accurately the elastic eigenfunctions and eigenfrequencies within a given frequency range (e.g. Pekeris \& Jarosch 1958; Woodhouse 1988; Dahlen \& Tromp 1998). Indeed, such calculations require the numerical solution of eigenvalue problems associated with systems of ordinary differential equations in the radial variable, and this can be done efficiently following the methods developed by Woodhouse (1988). Having obtained these eigenfunctions and eigenfrequencies, it is then a trivial matter to calculate theoretically complete synthetic seismograms using normal mode summation (e.g. Gilbert 1971). 
The Earth is not, of course, spherically symmetric, and most work in structural seismology over the past few decades has been focused on the inference of lateral variations in Earth's structure from seismic observations. To address such inverse problems, we must first be able to compute sufficiently accurate synthetic observations within a given laterally heterogeneous earth model. There now exist a range of methods - both asymptotic and exact - for undertaking these calculations, with the method of choice being dictated in large part by the frequency range of interest. In terms of fully numerical approaches we mention, in particular, the finite difference method (e.g. Boore 1972; Virieux 1986; Moczo 1989) and the spectral element method (e.g. Seriani et al. 1995; Faccioli et al. 1997; Komatitsch \& Vilotte 1998; Komatitsch \& Tromp 1999) that have made possible the calculation of accurate synthetic seismograms within laterally varying earth models over a wide range of frequencies, and have had a transformative effect on almost all aspects of seismology.

For studies of the Earth's free oscillations or tidal deformation, however, it is vital to account for self-gravitation and rotation. In fact, it is largely due to the effects of self-gravitation that sufficiently long-period deformations provide important constraints on the Earth's density structure. Lateral density variations remain poorly known within the Earth's mantle, but their robust determination will be vital in answering a range of important geodynamic questions (e.g. Ishii \& Tromp 1999; Trampert et al. 2004; Lay 2007; Moulik \& Ekström 2016; Lau et al. 2017). Modelling self-gravitation within fully numerical techniques such as the spectral element method is possible (Chaljub \& Valette 2004), but has not yet proven computationally feasible in practice. We note, however, that progress is being made in this area (Gharti \& Tromp 2017). Currently, the only viable method for modelling free oscillation spectra in a laterally heterogeneous earth model is normal mode coupling calculations that have been developed and refined over many years (e.g. Dahlen 1968, 1969; Woodhouse \& Dahlen 1978; Woodhouse 1980, 1983; Woodhouse \& Girnius 1982; Park \& Gilbert 1986; Romanowicz 1987; Lognonné \& Romanowicz 1990; Park 1990; Tromp \& Dahlen 1990; Hara et al. 1991, 1993; Lognonné 1991; Um \& Dahlen 1992; Deuss \& Woodhouse 2004; Al-Attar et al. 2012; Lau et al. 2015; Yang \& Tromp 2015). See Akbarashrafi et al. (2018) for a recent review of the underlying ideas.

Within normal mode coupling calculations, we start with a reference spherically symmetric earth model—typically the Premliminary Refernence Earth Model (PREM) of Dziewonski \& Anderson (1981) - for which the eigenfunctions and eigenfrequencies in a given frequency range can be calculated with relative ease. Let us initially assume that this spherically symmetric model is perturbed in such a way that its material parameters (i.e. elastic tensor and density) become laterally varying, but that its external and internal boundaries remain as concentric spheres. Such an earth model will be said to be 'geometrically spherical' in what follows. Within such a geometrically spherical earth model, we then wish to solve the elastodynamic equations. To do this, we expand the displacement field $\mathbf{u}$ in the form

$\mathbf{u}(\mathbf{x}, t)=\sum_{n} u_{n}(t) \mathbf{u}_{n}(\mathbf{x})$,

where the sum is taken over the infinitely many eigenfunctions $\mathbf{u}_{n}$ of the reference model, and the $u_{n}$ are time-dependent expansion coefficients to be determined. Such an expansion is possible because these reference eigenfunctions are assumed to form a complete basis for vector fields defined within the geometrically spherical earth model. That these eigenfunctions really do form a complete basis is known in the case of an entirely solid earth model, and can be inferred from general results in the theory of self-adjoint elliptic partial differential equations (e.g. Agmon et al. 1959; Browder 1961; Agmon 1962; Agmon et al. 1964; Thompson 1969; Simpson \& Spector 1987). Within earth models containing fluid regions, the associated spectral problem is significantly more complicated due to the occurrence of so-called undertone modes associated with density stratification in fluid regions (Valette 1989a,b; Rogister \& Valette 2009), and here the completeness property has not been definitively established. Nonetheless, it seems reasonable to assume that undertone modes are not of great importance for seismic applications as their eigenfunctions are essentially zero outside of fluid regions, and they appear to have periods well below that of interest in long-period seismology. Undertones may, however, be of greater importance when modelling tidal deformations or other long-period deformations such free core nutations or the Slichter mode.

Putting aside these technical issues, we assume that the eigenfunction expansion in eq. (1) is indeed exact, and in this manner the elastodynamic equations are reduced to determining the time-evolution of the expansion coefficients $u_{n}$. In the absence of lateral heterogeneities, the $u_{n}$ coefficients are completely decoupled from one another, and undergo forced simple harmonic motion at their particular eigenfrequency. The inclusion of lateral heterogeneity acts to couple the evolution equations satisfied by the expansion coefficients, and this results in a system of ordinary differential equations of the form

$\mathbf{M u ̈}+\mathbf{H u}=\mathbf{f}$,

where $\mathbf{u}$ is here an infinite-dimensional vector of the expansion coefficients, we use dots to denote time-derivatives, $\mathbf{M}$ is a 'mass-matrix' for the problem, $\mathbf{H}$ is a 'stiffness-matrix' and $\mathbf{f}$ is a force vector determined by the seismic source. Within this brief outline, we have chosen to neglect rotation and viscoelasticity in the problem, but these can be accommodated without difficulty into the full theory.

The coupled evolution equations in eq. (2) for the expansion coefficients are exact (given our assumption of completeness), but involve infinite-dimensional vectors and matrices. In practice, of course, we cannot work with such terms, and instead need to truncate the eigenfunction expansion in eq. (1) to include only finitely many terms. Crucially, however, these coupling calculations can be made as accurate as desired by including sufficiently many terms within the eigenfunction expansion. From this perspective it is, therefore, valid to say that mode coupling in geometrically spherical earth models provides an exact solution of the elastodynamic equations. Equally, the same is true for spectral element methods in the absence of self-gravitation, as here the solutions can be made arbitrarily accurate through refinement of the spatial and temporal discretization employed. We note that a recent paper of Akbarashrafi et al. (2018) has investigated practical convergence criteria 
for mode coupling calculations, and concluded that it is necessary to couple modes lying well beyond the frequency range of interest in order to obtain appropriate levels of accuracy.

The Earth is not geometrically spherical, and so we must also take account of the effects of surface topography along with topography on internal boundaries such as the core mantle boundary (CMB). Here, in particular, we mention the elliptical flattening of the Earth which has a pronounced effect on long-period free oscillation spectra. Within such geometrically aspherical earth models, however, the eigenfunction expansion in eq. (1) cannot be made for the simple reason that the reference eigenfunctions are not defined on the correct domain. To date, the effects of aspherical boundary topography have been included in normal mode coupling calculations using first-order boundary perturbation theory (Woodhouse \& Dahlen 1978; Woodhouse 1980). It would, of course, be preferable to have a method for these calculations that does not rely on such an approximation. Indeed, recent work has shown that other common approximations made within mode coupling calculations can lead to significant errors within synthetic spectra (e.g. Deuss \& Woodhouse 2001; Al-Attar et al. 2012; Yang \& Tromp 2015; Akbarashrafi et al. 2018), and such errors must feed through into Earth models obtained by solution of the corresponding inverse problem-see Valentine \& Trampert (2015) for a discussion of the impact of such theoretical errors on inverse problems. Furthermore, in the coming years it seems likely that efficient fully numerical methods will be developed for calculating long-period free oscillation spectra. Whether such methods will supersede mode coupling calculations is difficult to judge, and ultimately will depend on the relative computational cost of the different approaches. It is, however, certain that such fully numerical methods will be able to model boundary topography without approximation, and so the incorporation of this feature is highly desirable within mode coupling calculations going forward.

A way to account for boundary topography exactly within normal mode coupling calculations was suggested within Woodhouse (1976). The idea is very simple; we introduce a mapping from a geometrically spherical reference earth model onto the geometrically aspherical model of interest. Using this mapping, we can then perform a 'change of variables' in the equations of motion, and so reformulate them as an equivalent system of equations within the geometrically spherical reference body. Having done this, the eigenfunction expansion in eq. (1) can then be performed without approximation, and the coupling calculations proceed in the same manner as discussed above. The difficult step in this process is transforming the equations of motion under such a mapping. Woodhouse (1976) carried out the necessary calculations partially, but his work was focused on developing of a suitable boundary perturbation theory, and not on mode coupling as such. We note that Jobert (1976) and Cisternas \& Jobert (1977) applied similar coordinate transformation to account for boundary topography in layer-matrix type calculations. Takeuchi (2005) later showed how these transformations could be performed exactly for linear elasticity within a Cartesian geometry and in the absence of self-gravitation. Within AC16, we developed a general theory for such transformations within both finite and linearized elasticity and also incorporated self-gravitation and viscoelasticity into the problem. Furthermore, AC16 made a connection between these ideas and work within applied mathematics and physics literature related to cloaking, invisibility and meta-materials (e.g. Stefanov \& Uhlmann 1998a,b; Greenleaf et al. 2003; Mazzucato \& Rachele 2006; Pendry et al. 2006; Rahm et al. 2008). In this paper, we show how the particle relabelling transformations introduced in AC16 can be extended to the case of a planet that contains both fluid and solid regions. As will be seen, this extension to fluid-solid bodies is non-trivial due to the occurrence of tangential slip at fluid--solid boundaries, and the main the challenges involved are outlined in the second part of this introduction which shifts to a more technical tone. Readers interested only in the application of the final results to mode coupling calculations are encouraged now to jump ahead to Section 4.

\subsection{Reference configurations for an elastic solid}

To understand the work of AC16 on particle relabelling transformations, it will be useful to review how the motion of a solid elastic body is described mathematically. Along the way, we will recall some necessary terminology for later reference (for additional background see, e.g. Abraham et al. 2012). Note that we consider here finite deformations of the body, meaning that there is no assumption that the motion is 'small' in any sense. Even though most geophysical applications are concerned only with linearized deformations about equilibrium, it is preferable to start from an exact theory, and so retain the geometric insight into the problem provided. Having taken this approach, reduction to the case of linearized motions requires only simple, if tedious, calculations, and we can be sure that no relevant physics has been inadvertently neglected.

To describe the motion of such an elastic solid, we first select an inertial reference frame, and so identify physical space with $\mathbb{R}^{3}$ and fix an origin time. At time $t$, the body will occupy a subset $M_{t} \subseteq \mathbb{R}^{3}$. We assume that $M_{t}$ is compact, has a connected and open interior, along with a smooth boundary $\partial M_{t}$. An example of such a subset is the closed unit ball $\left\{\mathbf{x} \in \mathbb{R}^{3} \mid\|\mathbf{x}\|=1\right\}$, and we note these technical conditions simply ensure that the body has a physically sensible shape. At a different time $t^{\prime} \neq t$, the body will, in general, occupy a different subset $M_{t^{\prime}} \subseteq \mathbb{R}^{3}$. We shall not allow motions of the body in which it is torn or fractured, and so the two subsets $M_{t}$ and $M_{t^{\prime}}$ must always be homeomorphic. This means that there exists a continuous mapping from $M_{t}$ onto $M_{t^{\prime}}$ that has a continuous inverse. In fact, we shall impose the stronger requirement that these mappings are smooth-i.e. that they have continuous partial derivatives of all orders-and so are said to be diffeomorphisms, and the two sets $M_{t}$ and $M_{t^{\prime}}$ to be diffeomorphic.

At the time $t$, we consider the particle of the body that is instantaneously located at the point $\mathbf{y} \in M_{t}$, with the term 'particle' here being used by analogy with classical mechanics. As time progresses, this particle will move along a smooth curve in $\mathbb{R}^{3}$ and by time $t^{\prime}$ will have arrived at some new location $\mathbf{y}^{\prime} \in M_{t^{\prime}}$. In this manner, the motion of the body is associated with a unique diffeomorphism mapping $M_{t}$ onto $M_{t^{\prime}}$. This correspondence could be used to identify the particles of the body with parametrized curves in $\mathbb{R}^{3}$, with this idea closely resembling the world lines of relativistic physics. This invariant point of view is not practically useful, however, and we instead require a concrete method 
for labelling the particles within the body. Such a labelling scheme defines a reference configuration for the body relative to which its motion can be described, and it is vital to appreciate that there are infinitely many ways this labelling can be carried out. For example, the most common way to set up a reference configuration is to label each particle of the body with its position vector $\mathbf{x} \in \mathbb{R}^{3}$ at the origin time (e.g. Dahlen \& Tromp 1998, section 2.1). Another frequently used approach is to label particles by their position vector when the body is in a state of equilibrium, with this choice being useful in linearized problems where the equilibrium positions are known a priori. Within a given application we are, however, always free to choose the labelling scheme that seems most convenient. In particular, there is absolutely no reason why the reference configuration needs to be one the body ever actually inhabits (Antman 2005, chapter 12).

In whatever manner the particle labelling is done, it results in a reference body $M \subseteq \mathbb{R}^{3}$ which is again compact, with open and connected interior, and smooth boundary $\partial M$. The elements of $M$ correspond in a one-to-one manner with particles in the body, and the motion relative to this reference configuration is described by a smooth mapping $\varphi: M \times \mathbb{R} \rightarrow \mathbb{R}^{3}$ such that $\varphi(\mathbf{x}, t) \in \mathbb{R}^{3}$ is the position at time $t$ of the particle labelled by $\mathbf{x} \in M$. For each fixed time $t$, we denote by $\varphi(\cdot, t)$ the mapping $M \ni \mathbf{x} \mapsto \varphi(\mathbf{x}, t) \in M_{t} \subseteq \mathbb{R}^{3}$, which we call the instantaneous configuration of the body. The instantaneous configuration $\varphi(\cdot, t): M \rightarrow \mathbb{R}^{3}$ is not a diffeomorphism because it does not map $M$ onto the whole of $\mathbb{R}^{3}$, and hence its inverse is not always defined. If, however, we regard $\varphi(\cdot, t)$ as a mapping from $M$ onto its image $M_{t}=\varphi(M, t)$, then it becomes a diffeomorphism. Mappings with this property are known as embeddings, and so we see that the configuration space of an elastic solid comprises all possible embeddings from the reference body $M$ into physical space $\mathbb{R}^{3}$. This infinite-dimensional configuration space is a natural generalization of the configuration spaces familiar within classical mechanics (e.g. Abraham et al. 1978). Indeed, even in such finite-dimensional problems we still need a scheme for labelling the different particles, and this can be done in several ways. In what follows, it will be useful to write $\operatorname{Emb}\left(M ; \mathbb{R}^{3}\right)$ for the set of all smooth embeddings of $M$ into $\mathbb{R}^{3}$. For later reference, we note that embeddings can be similarly defined from surfaces or curves into $\mathbb{R}^{3}$. For example, consider the restriction of the instantaneous configuration $\varphi(\cdot, t)$ to the boundary $\partial M$ of $M$ which we denoted by $\left.\varphi(\cdot, t)\right|_{\partial M}: \partial M \rightarrow \mathbb{R}^{3}$. This restricted mapping defines an embedding of $\partial M$ into $\mathbb{R}^{3}$ because it induces a diffeomorphism from $\partial M$ onto its image $\partial M_{t}$ (here one strictly needs to appreciate that $\partial M$ and $\partial M_{t}$ are smooth manifolds, and that diffeomorphisms can be defined between smooth manifolds in a natural manner).

\subsection{Particle relabelling transformations in an elastic solid}

We have seen that a reference configuration is necessary to describe the motion of an elastic solid, and that each such reference configuration corresponds to a scheme for labelling the particles within the body. Suppose such a reference configuration has been established for a body, and that its motion relative to this reference configuration is given by the mapping $\varphi: M \times \mathbb{R} \rightarrow \mathbb{R}^{3}$, where $M$ is the associated reference body. This same motion can, of course, be equivalently described relative to some other reference configuration, and hence by a mapping $\tilde{\varphi}: \tilde{M} \times \mathbb{R} \rightarrow \mathbb{R}^{3}$, where $\tilde{M}$ is a generally distinct reference body. These two mappings represent the same physical process, and so there must be a relationship between them. Particle relabelling transformations were introduced by AC16 precisely to answer this question. Each point $\mathbf{x} \in M$ labels a particle within the body, and this particle is equivalently labelled by a unique point $\tilde{\mathbf{x}} \in \tilde{M}$. This one-to-one correspondence establishes a unique diffeomorphism $\xi: \tilde{M} \rightarrow M$ between the two reference bodies such that

$\mathbf{x}=\xi(\tilde{\mathbf{x}})$.

The diffeomorphism $\xi: \tilde{M} \rightarrow M$ is what is meant by particle relabelling transformation, and its effect is indeed simply to change the labelling scheme used to describe the particles of the body. Given eq. (3), it is clear that the two motions $\varphi$ and $\tilde{\varphi}$ must be related through

$\tilde{\varphi}(\mathbf{x}, t)=\varphi[\xi(\mathbf{x}), t]$

for all $(\mathbf{x}, t) \in \tilde{M} \times \mathbb{R}$. It is important to note that particle relabelling transformations can act between reference configurations having a common reference body $M$, and so the reference configuration is not synonymous with the reference body (this point was not always made as clearly in AC16 as it should have been). In such cases, the particle relabelling transformations are diffeomorphisms from $M$ onto itself, and it is readily seen that these mappings form a group under composition. This group, which will be needed below, is denoted by Diff( $M)$, is known as the diffeomorphism group of $M$ (e.g. Ebin \& Marsden 1970; Omori 1970, 1974). Again, we note that a diffeomorphism group can also be defined for smooth manifolds such as $\partial M$. Within AC16, the key results obtained were expressions showing how the material parameters (referential density and strain energy function) of a body described relative to different reference configurations are related. The derivation of these results was greatly simplified through use of an argument based on Hamilton's principle, and this allowed us to circumvent the lengthy and intricate calculations present in the related work of Mazzucato \& Rachele (2006).

\subsection{Extension of particle relabelling transformations to fluid-solid bodies}

Much of the preceding discussion carries over directly to fluid-solid bodies. Here, however, there exist non-trivial complications associated with tangential slip across fluid-solid boundaries. We will not enter into these issues fully within this introduction, but simply outline the difficulties along with our approach to their solution. Within seismology and other parts of solid earth geophysics, it is common to treat fluid regions of the Earth (e.g. the outer core) as being elastic fluids, meaning that they are compressible, but have no viscosity. As a result, these fluids are able to flow tangentially along their boundaries with adjacent solid regions (Dahlen 1972; Woodhouse \& Dahlen 1978). Due to the 
possibility of such tangential slip, the motion of the body need not be a smooth embedding, and the problem's configuration space must be suitably enlarged. We note that tangential slip of an inviscid fluid along a solid interface should be understood physically as an approximation to the thin boundary layers that would form within a real fluid having finite but low viscosity. It is for this reason that we still require the motion within the interior of fluid regions to be smooth.

As mentioned above, the analysis in AC16 of particle relabelling transformations was based on Hamilton's principle for finite elasticity. As a formulation of this principle within a fluid-solid finite elastic body was unknown to us, the first part of this paper shows how this can be done both through the use of constrained variations and by introducing a suitable Lagrangian multiplier field. We note that during the preparation of this work an alternative formulation of Hamilton's principle for a fluid-solid body has been presented in a pre-print by Brazda et al. (2017). These authors, however, fix a choice of reference configuration for the problem, and so their approach lacks sufficient generality for our purposes.

Having obtained the necessary form of Hamilton's principle, we show that the approach of AC16 to particle relabelling transformations carries over in a simple manner to fluid-solid bodies. Furthermore, we obtain an associated variational principle for the linearized equations of motion required in applications to long-period seismology, tidal deformation and other related fields. These results generalize the first complete discussion of this problem given by Woodhouse \& Dahlen (1978), and reduce to a form equivalent to theirs for the standard choice of reference configuration. We note that Woodhouse \& Dahlen (1978) also showed that the linearized equations of motion could be obtained from a variational principle, but here they incorporated surface integral terms into their action in an ad hoc manner to reproduce the necessary continuity conditions at fluid-solid boundaries they had obtained through an application of Newton's third law of motion. In contrast, we obtain analogous surface integral contributions within our action for the linearized problem in a direct and clear manner from the exact variational principle, and so gain insight into the origin of these terms.

\section{HAMILTON'S PRINCIPLE FOR FINITE ELASTICITY}

\subsection{Vector and tensor notation}

Here we explain some notations for future reference. We use boldface type for vectors, tensors and associated linear operators. The action of a linear operator $\mathbf{A}$ on an appropriate vector $\mathbf{u}$ will always be written in the form $\mathbf{A} \cdot \mathbf{u}$. The composition of two linear operators $\mathbf{A}$ and $\mathbf{B}$ will be indicated by their juxtaposition, and so we have $(\mathbf{A B}) \cdot \mathbf{u}=\mathbf{A} \cdot(\mathbf{B} \cdot \mathbf{u})$ for any suitable vector $\mathbf{u}$. For two tensors $\mathbf{T}$ and $\mathbf{S}$ of order $k$, we define their inner product

$\langle\mathbf{T}, \mathbf{S}\rangle=T_{i_{1} \cdots i_{k}} S_{i_{1} \cdots i_{k}}$,

where the summation convention is being applied on the right-hand side. The norm of such tensors is then given by

$\|\mathbf{T}\|=\sqrt{\langle\mathbf{T}, \mathbf{T}\rangle}$.

Note that the same symbols are used for the inner product and norm of all tensors irrespective of their order, but with sufficient care this should not lead to any confusion. If $\mathbf{A}$ is a linear operator mapping tensors of order $k$ into tensors of order $l$, then its transpose $\mathbf{A}^{T}$ is the unique linear operator mapping tensors of order $l$ into tensors of order $k$ defined through

$\langle\mathbf{A} \cdot \mathbf{T}, \mathbf{S}\rangle=\left\langle\mathbf{T}, \mathbf{A}^{T} \cdot \mathbf{S}\right\rangle$,

for all suitable $\mathbf{T}$ and $\mathbf{S}$. In terms of components, it is clear that the transpose of $\mathbf{A}$ is given by

$\left(\mathbf{A}^{T}\right)_{j_{1} \cdots j_{k} i_{1} \cdots i_{l}}=A_{i_{1} \cdots i_{l} j_{1} \cdots j_{k}}$.

\subsection{Applications to a solid body}

We begin by recalling Hamilton's principle for a solid finite elastic body as this will establish notations, and also motivate our approach to the fluid-solid problem. This discussion will follow closely section 2 of AC16, which is, in turn, based on standard material within the continuum mechanics and theoretical geophysics literature including the works of Noll (1974), Marsden \& Hughes (1994), Dahlen \& Tromp (1998), Holzapfel (2002), Truesdell \& Noll (2004), Antman (2005) and Woodhouse \& Deuss (2007). For the most part we retain the notations of $\mathrm{AC16}$, but in a few places refinements have been introduced to improve the clarity of presentation.

\subsubsection{Kinematics}

As discussed in Section 1, to describe the motion of a solid elastic body we need to select a suitable reference configuration. Importantly, however, we note that the form of the resulting equations of motion is completely independent of this choice, and so there is no need to describe explicitly which reference configuration is used for the moment. We write $M \subseteq \mathbb{R}^{3}$ for the associated reference body, and denote the motion relative to the reference configuration by the smooth mapping $\varphi: M \times I \rightarrow \mathbb{R}^{3}$, where $I=[0, T]$ is the time interval of interest. As above, we assume that the instantaneous configurations $\varphi(\cdot, t): M \rightarrow \mathbb{R}^{3}$ for each $t \in I$ are embeddings, though later we outline the slight 
modification necessary if there exist internal boundaries within the body across which the material parameters fail to be smooth. From the motion, we need to introduce a number of derived quantities. First, there is the referential velocity $\mathbf{v}=\partial_{t} \boldsymbol{\varphi}$, whose value at $(\mathbf{x}, t) \in M \times I$ represents the velocity of the particle labelled by $\mathbf{x} \in M$ at time $t$, when it is located at the spatial point $\varphi(\mathbf{x}, t)$. Next, we have the deformation gradient of the motion $\mathbf{F}=\operatorname{Def} \varphi$, where the action of the deformation operator, Def, on the motion is defined invariantly such that

$\varphi(\mathbf{x}+\delta \mathbf{x}, t)=\varphi(\mathbf{x}, t)+(\operatorname{Def} \varphi)(\mathbf{x}, t) \cdot \delta \mathbf{x}+o\left(\|\delta \mathbf{x}\|^{2}\right)$,

or equivalently in terms of components as

$(\operatorname{Def} \varphi)_{i j}=\frac{\partial \varphi_{i}}{\partial x_{j}}$

The Jacobian of the motion $J=\operatorname{det} \mathbf{F}$ relates a volume element $\mathrm{d}^{3} \mathbf{x}$ within the reference body to the corresponding volume element $\mathrm{d}^{3} \mathbf{y}$ in physical space through the change of variables formula

$\mathrm{d}^{3} \mathbf{y}=|J(\mathbf{x}, t)| \mathrm{d}^{3} \mathbf{x}$.

In order for the instantaneous configurations $\varphi(\cdot, t)$ to be embeddings, it follows from the inverse function theorem (e.g. Abraham et al. 2012) that $J$ can never vanish, and so must always and everywhere be of one sign. Without loss of generality, we shall assume that $J>0$, in which case the motion is said to be orientation preserving, and we can drop the absolute value sign from eq. (11). Note further that all embeddings or diffeomorphisms will be implicitly assumed to be orientation preserving in what follows.

\subsubsection{Mass conservation and gravitational potential}

During the body's motion, the mass of any of its subregions must be conserved. We write $\varrho(\mathbf{y}, t)$ for the density of the body at the spatial point $\mathbf{y} \in M_{t}$ and time $t$. Let $U \subseteq M$ be an open subset of the reference body, and $U_{t}=\varphi(U, t)$ its image under the motion at time $t$. The mass of this subregion is given by $\int_{U_{t}} \varrho(\mathbf{y}, t) \mathrm{d}^{3} \mathbf{y}$, and the time-derivative of this quantity must vanish. We can use the instantaneous configuration $\varphi(\cdot, t)$ to transform the integration to be over $U \subseteq M$, and so obtain

$\int_{U} \frac{\partial}{\partial t}\{J(\mathbf{x}, t) \varrho[\varphi(\mathbf{x}, t), t]\} \mathrm{d}^{3} \mathbf{x}=0$.

As the subregion $U$ is arbitrary, we conclude that the integrand vanishes identically, and so are led to introduce the body's referential density as defined by

$\rho(\mathbf{x})=J(\mathbf{x}, t) \varrho[\varphi(\mathbf{x}, t), t]$.

This referential density is regarded as a material parameter of the body (relative to the chosen reference configuration), and from its value along with knowledge of the motion, we can use eq. (13) to determine the density of the body at any spatial point and time.

For applications to long-period seismology and related fields, self-gravitation forms an essential component of elastodynamics. The gravitational potential of the body at the instant in time $t$ is given by the familiar expression

$\phi(\mathbf{y}, t):=\int_{M_{t}} \varrho\left(\mathbf{y}^{\prime}, t\right) \Gamma\left(\mathbf{y}-\mathbf{y}^{\prime}\right) \mathrm{d}^{3} \mathbf{y}^{\prime}$,

where $\Gamma$ is the Newtonian potential

$\Gamma(\mathbf{y})=-\frac{G}{\|\mathbf{y}\|}$

with $G$ the universal gravitational constant. It can also be shown (e.g. Dahlen \& Tromp 1998, section 2.9) that the body's instantaneous gravitational binding energy at a time $t$ is equal to

$\frac{1}{2} \int_{M_{t}} \varrho(\mathbf{y}, t) \phi(\mathbf{y}, t) \mathrm{d}^{3} \mathbf{y}$

To include gravitational forces into Hamilton's principle, it is useful to work with quantities defined only on the reference body. First, we define the referential gravitational potential of the body to be

$\zeta(\mathbf{x}, t):=\phi[\varphi(\mathbf{x}, t), t]=\int_{M} \rho\left(\mathbf{x}^{\prime}\right) \Gamma\left[\varphi(\mathbf{x}, t)-\varphi\left(\mathbf{x}^{\prime}, t\right)\right] \mathrm{d}^{3} \mathbf{x}^{\prime}$,

for $(\mathbf{x}, t) \in M \times I$, where in obtaining the second equality we used $\varphi(\cdot, t)$ to change variables within the volume integral, and have made use of eq. (13). Note that knowledge of $\zeta$ and the motion $\varphi$ is sufficient to determine $\phi$ in all space. This is because the gravitational potential exterior to the instantaneous body $M_{t}$ is uniquely determined by its values on $\partial M_{t}$. From this definition, it is then easy to see that the instantaneous gravitational binding energy can be written in the form

$\frac{1}{2} \int_{M} \rho(\mathbf{x}) \zeta(\mathbf{x}, t) \mathrm{d}^{3} \mathbf{x}$,

which does indeed depend only on referential quantities. 


\subsubsection{Equations of motion and Hamilton's principle}

There are a number of different methods for obtaining the equations of motion of finite elasticity in a solid body, with perhaps the most elementary being the integral balance laws for linear and angular momentum (e.g. Dahlen \& Tromp 1998, section 2.7). For the analysis of particle relabelling transformations, however, we have found it more convenient to work with Hamilton's principle of stationary action. By doing so, we need only consider the transformation properties of a scalar-valued action, and so avoid most of the intricacies with tensor-valued quantities and associated differential operators. This variational principle is, however, only applicable within so-called hyperelastic bodies that have a well-defined elastic potential energy associated with changes to their shape. As a result, we are not able to consider dissipative mechanisms that can sometimes be important. The extension of particle relabelling transformations to finite viscoelastic bodies has been outlined in an appendix to AC16, and so our present focus on hyperelastic bodies does not represent a fundamental limitation. Furthermore, we restrict attention to the case of simple hyperelastic bodies, these being ones whose elastic potential energy depends on the motion in a local manner, and through only its first-order spatial derivatives (i.e. the deformation gradient). The need to consider higher-order or non-local formulations of hyperelasticity within geophysics has, to our knowledge, never been proposed.

For a simple hyperelastic body, the elastic potential energy associated with changes of its shape can be written in the following form

$\int_{M} W[\mathbf{x}, \mathbf{F}(\mathbf{x}, t)] \mathrm{d}^{3} \mathbf{x}$

where $W$ is known as the strain energy function of the the body, and we recall that $\mathbf{F}=\operatorname{Def} \boldsymbol{\varphi}$ is the deformation gradient associated with the motion. We note that this elastic potential energy shows no explicit dependence on the motion itself, with this fact reflecting the required invariance under superimposed rigid body translations. Similarly, the elastic potential energy has no explicit dependence on time, and must also be invariant under all superimposed rigid body rotations. These three properties of the elastic potential energy constitute the appropriate form of the principle of material frame indifference (e.g. Truesdell \& Noll 2004) for simple hyperelastic bodies. To insure that the elastic potential energy is indeed invariant under all superimposed rigid body rotations, it may be shown using the polar decomposition theorem (e.g. Marsden \& Hughes 1994) that the strain energy function can only depend on the deformation gradient in terms of the right Cauchy-Green deformation tensor $\mathbf{C}=\mathbf{F}^{T} \mathbf{F}$, and hence that this strain energy function takes the form

$W(\mathbf{x}, \mathbf{F})=U(\mathbf{x}, \mathbf{C})$,

where $U$ is some auxiliary function. Within AC16, it was shown that a stress-glut representation of a seismic source (Backus \& Mulcahy 1976) can be added into the problem by allowing the strain energy function to have the explicit time-dependence

$W(\mathbf{x}, t, \mathbf{F})=U(\mathbf{x}, \mathbf{C})-\frac{1}{2}\langle\mathfrak{S}(\mathbf{x}, t), \mathbf{C}\rangle$,

where the stress glut $\mathfrak{S}$ is a symmetric second-order tensor field.

Given the above definitions, we can now write down the action for a self-gravitating simple hyperelastic solid in the usual form

$\mathcal{S}:=\int_{I} \int_{M}\left\{\frac{1}{2} \rho(\mathbf{x})\|\mathbf{v}(\mathbf{x}, t)\|^{2}-W[\mathbf{x}, t, \mathbf{F}(\mathbf{x}, t)]-\frac{1}{2} \rho(\mathbf{x}) \zeta(\mathbf{x}, t)\right\} \mathrm{d}^{3} \mathbf{x} \mathrm{d} t$,

where the Lagrangian density (i.e. the integrand) is given by the kinetic energy density minus potential energy terms associated both with elastic strain energy and the body's gravitational binding energy. Such an action is well known within the continuum mechanics literature (e.g. Marsden \& Hughes 1994), though we note that the inclusion of self-gravitation into the problem is due to Woodhouse \& Deuss (2007). This action is a function of the body's motion, meaning that for each motion $\varphi$ it returns a single real number $\mathcal{S}[\varphi]$. We note, however, the action depends on the given motion implicitly through the associated referential velocity $\mathbf{v}$, deformation gradient $\mathbf{F}$ and referential gravitational potential $\zeta$ as defined previously. It is also worth noting that Woodhouse \& Deuss (2007) handled gravitational terms in a different way, with these authors incorporating the gravitational potential $\phi$ as an independent field within the variational problem via the method of Lagrangian multipliers. We have, however, found it simpler to work with the referential gravitational potential $\zeta$, and to regard it as a function of the motion as given by eq. (17).

To obtain the equations of motion for the body from Hamilton's principle, suppose we have been given the following initial conditions on the motion $\varphi$ and the associated referential velocity $\mathbf{v}$ :

$\varphi(\mathbf{x}, 0)=\boldsymbol{\Phi}(\mathbf{x}), \quad \mathbf{v}(\mathbf{x}, 0)=\mathbf{V}(\mathbf{x})$.

Let $\bar{\varphi}: M \times I \times J \rightarrow \mathbb{R}^{3}$ be a smooth one-parameter family of motions indexed by $\epsilon$ defined in some open neighbourhood $J$ of 0 , and satisfying the following conditions

$\bar{\varphi}(\mathbf{x}, t, 0)=\varphi(\mathbf{x}, t)$

$\bar{\varphi}(\mathbf{x}, 0, \epsilon)=\boldsymbol{\Phi}(\mathbf{x}), \quad \overline{\mathbf{v}}(\mathbf{x}, 0, \epsilon)=\mathbf{V}(\mathbf{x})$,

$\partial_{\epsilon} \bar{\varphi}(\mathbf{x}, T, \epsilon)=\mathbf{0}, \quad \partial_{\epsilon} \overline{\mathbf{v}}(\mathbf{x}, T, \epsilon)=\mathbf{0}$,

for all possible values of the arguments, and where we recall that $I=[0, T]$ is the time-interval of interest. The first condition means that $\epsilon=0$ corresponds to the actual motion $\varphi$ of the body, while the remaining conditions require that all motions within the one-parameter family 
satisfy the initial conditions and have the same terminal conditions. Such a one-parameter family $\bar{\varphi}$ is known as a variation about $\varphi$, and we can use it to form the function

$\epsilon \mapsto \mathcal{S}[\bar{\varphi}(\cdot, \cdot, \epsilon)]$,

where the right-hand side denotes the value of the action for the motion $\bar{\varphi}(\cdot, \cdot, \epsilon): M \times I \rightarrow \mathbb{R}^{3}$. Hamilton's principle requires vanishing of the first variation of the action

$$
\left.\frac{\mathrm{d}}{\mathrm{d} \epsilon} \mathcal{S}[\bar{\varphi}(\cdot, \cdot, \epsilon)]\right|_{\epsilon=0}=0
$$

for all possible variations about $\varphi$. The Euler-Lagrange equations and natural boundary conditions associated with this variational principle give the desired equations of motion for a simple hyperelastic body. A full derivation of these equations can be found within in section 2.5 of $\mathrm{AC} 16$, and leads to the familiar momentum equation

$\rho \partial_{t} \mathbf{v}-\operatorname{Div} \mathbf{T}-\rho \boldsymbol{\gamma}=\mathbf{0}$,

which is to hold for $(\mathbf{x}, t) \in M \times I$, where

$\mathbf{T}(\mathbf{x}, t)=\frac{\partial W}{\partial \mathbf{F}}[\mathbf{x}, t, \mathbf{F}(\mathbf{x}, t)]$,

is the first Piola-Kirchhoff stress tensor whose divergence is defined as

$(\operatorname{Div} \mathbf{T})_{i}:=\partial_{j} T_{i j}$,

and $\gamma$ is the referential gravitational acceleration

$\boldsymbol{\gamma}(\mathbf{x}, t):=-\int_{M} \rho\left(\mathbf{x}^{\prime}\right)(\nabla \Gamma)\left[\varphi(\mathbf{x}, t)-\varphi\left(\mathbf{x}^{\prime}, t\right)\right] \mathrm{d}^{3} \mathbf{x}^{\prime}$.

These equations are subject to the boundary condition

$\mathbf{t}(\mathbf{x}, t)=\mathbf{T}(\mathbf{x}, t) \cdot \hat{\mathbf{n}}(\mathbf{x})=\mathbf{0}$,

for all $(\mathbf{x}, t) \in \partial M \times I$ where $\hat{\mathbf{n}}$ is the outward unit normal vector, and $\mathbf{t}$ denotes the referential traction vector on $\partial M$.

In obtaining these results we have, however, assumed that the material parameters $\rho$ and $W$ depend smoothly on $\mathbf{x} \in M$. If, instead, these parameters fail to be smooth, then our approach requires slight modification. The situation of greatest practical interest is when $\rho$ and $W$ are piecewise-smooth, with their discontinuities forming closed, smooth, non-intersecting, 2-D surfaces within the body. Across each such solid-solid boundary the motion must be continuous, but we cannot assume that it will have continuous spatial derivatives. We must, therefore, enlarge the configuration space of possible motions to include piecewise-smooth embeddings that are continuous across solid-solid boundaries. Having done this, derivation of the equations of motion proceeds largely as before, except we now obtain the familiar condition that the referential traction is continuous across each solid-solid boundary. Because solid-solid boundaries can be handled in this straightforward manner, we shall leave their presence implicit in what follows to simplify the presentation.

\subsubsection{Motion relative to a steadily rotating reference frame}

The equations of motion obtained in Section 2.2 are defined relative to an inertial reference frame. For applications in global seismology or geodesy, it is often more convenient to use a non-inertial reference frame that approximately corotates with the Earth. As we consider only internal forces acting on the earth model, we can select an inertial reference frame relative to which the body's centre of mass is fixed at the origin. We can then introduce a non-inertial frame undergoing a steady rotation about an axis passing through the centre of mass. Such a rotating frame is related to the inertial frame by a rotation matrix $\mathbf{R}(t)$ (i.e. orthogonal with unit determinant). We then define the associated angular velocity matrix

$\hat{\mathbf{\Omega}}=\mathbf{R}(t)^{T} \frac{\mathrm{d} \mathbf{R}}{\mathrm{d} t}(t)$,

which is readily seen to be antisymmetric, and is constant by definition for a steady rotation. Without loss of generality, we assume that $\mathbf{R}(0)=\mathbf{1}$. Relative to the inertial reference frame, we can then decompose the motion in the form

$\varphi(\mathbf{x}, t)=\mathbf{R}(t) \cdot \varphi_{b}(\mathbf{x}, t)$,

where $\varphi_{b}: M \times I \rightarrow \mathbb{R}^{3}$ represents the internal motion with respect to the steadily rotating reference frame. A simple calculation using eq. (34) shows that

$\mathbf{v}(\mathbf{x}, t)=\mathbf{R}(t) \cdot\left[\mathbf{v}_{b}(\mathbf{x}, t)+\hat{\mathbf{\Omega}} \cdot \boldsymbol{\varphi}_{b}(\mathbf{x}, t)\right], \quad \mathbf{F}(\mathbf{x}, t)=\mathbf{R}(t) \mathbf{F}_{b}(\mathbf{x}, t)$,

where we have defined

$\mathbf{v}_{b}(\mathbf{x}, t)=\left(\partial_{t} \varphi_{b}\right)(\mathbf{x}, t), \quad \mathbf{F}_{b}(\mathbf{x}, t)=\left(\operatorname{Def} \boldsymbol{\varphi}_{b}\right)(\mathbf{x}, t)$. 
As is well known (e.g. Holm et al. 2009), there exists a unique angular velocity vector $t \mapsto \boldsymbol{\Omega} \in \mathbb{R}^{3}$ such that for any a $\in \mathbb{R}^{3}$ we have $\hat{\boldsymbol{\Omega}} \cdot \mathbf{a}=\boldsymbol{\Omega} \times \mathbf{a}$, and so we can write the above expression for the velocity relative to the inertial reference frame as

$\mathbf{v}(\mathbf{x}, t)=\mathbf{R}(t) \cdot\left[\mathbf{v}_{b}(\mathbf{x}, t)+\mathbf{\Omega} \times \boldsymbol{\varphi}_{b}(\mathbf{x}, t)\right]$.

Substituting these expressions into the action in eq. (22), we obtain

$\mathcal{S}[\boldsymbol{\varphi}]=\int_{I} \int_{M}\left\{\frac{1}{2} \rho(\mathbf{x})\left\|\mathbf{v}_{b}(\mathbf{x}, t)+\boldsymbol{\Omega} \times \boldsymbol{\varphi}_{b}(\mathbf{x}, t)\right\|^{2}-W\left[\mathbf{x}, t, \mathbf{F}_{b}(\mathbf{x}, t)\right]-\frac{1}{2} \rho(\mathbf{x}) \zeta_{b}(\mathbf{x}, t)\right\} \mathrm{d}^{3} \mathbf{x} \mathrm{d} t$,

where the referential gravitational potential associated with the internal motion is given by

$\zeta_{b}(\mathbf{x}, t):=\int_{M} \rho\left(\mathbf{x}^{\prime}\right) \Gamma\left[\varphi_{b}(\mathbf{x}, t)-\boldsymbol{\varphi}_{b}\left(\mathbf{x}^{\prime}, t\right)\right] \mathrm{d}^{3} \mathbf{x}^{\prime}$,

with the subscript added to $\zeta_{b}$ for notational symmetry, and we have used

$W\left[\mathbf{x}, t, \mathbf{R}(t) \mathbf{F}_{b}(\mathbf{x}, t)\right]=W\left[\mathbf{x}, t, \mathbf{F}_{b}(\mathbf{x}, t)\right]$,

which follows from the principle of material frame indifference. As the value of the action does not depend explicitly on $\mathbf{R}$, it will be convenient to define a reduced action

$\mathcal{S}_{b}\left[\boldsymbol{\varphi}_{b}\right]:=\int_{I} \int_{M}\left\{\frac{1}{2} \rho(\mathbf{x})\left\|\mathbf{v}_{b}(\mathbf{x}, t)+\boldsymbol{\Omega} \times \boldsymbol{\varphi}_{b}(\mathbf{x}, t)\right\|^{2}-W\left[\mathbf{x}, t, \mathbf{F}_{b}(\mathbf{x}, t)\right]-\frac{1}{2} \rho(\mathbf{x}) \zeta_{b}(\mathbf{x}, t)\right\} \mathrm{d}^{3} \mathbf{x} \mathrm{d} t$,

which is a function of the internal motion $\varphi_{b}$ alone. In determining equations of motion associated with $\mathcal{S}_{b}$, we only need to consider the modified kinetic energy term whose first variation is given by

$\int_{I} \int_{M} \rho\left\langle\mathbf{v}_{b}+\boldsymbol{\Omega} \times \boldsymbol{\varphi}_{b}, \delta \mathbf{v}_{b}+\boldsymbol{\Omega} \times \delta \boldsymbol{\varphi}_{b}\right\rangle \mathrm{d}^{3} \mathbf{x} \mathrm{d} t$,

and, on integration by parts, this can be written as

$-\int_{I} \int_{M} \rho\left\langle\partial_{t} \mathbf{v}_{b}+2 \boldsymbol{\Omega} \times \mathbf{v}_{b}+\boldsymbol{\Omega} \times\left(\boldsymbol{\Omega} \times \boldsymbol{\varphi}_{b}\right), \delta \boldsymbol{\varphi}_{b}\right\rangle \mathrm{d}^{3} \mathbf{x} \mathrm{d} t$.

It follows that the momentum equation relative to the rotating reference frame is given by

$\rho \partial_{t} \mathbf{v}_{b}+2 \rho \boldsymbol{\Omega} \times \mathbf{v}_{b}+\rho \boldsymbol{\Omega} \times\left(\boldsymbol{\Omega} \times \boldsymbol{\varphi}_{b}\right)-\operatorname{Div}_{b}-\rho \boldsymbol{\gamma}_{b}=\mathbf{0}$,

where $\mathbf{T}_{b}$ and $\boldsymbol{\gamma}_{b}$ are, respectively, the first Piola-Kirchhoff stress tensor and referential gravitational acceleration of the internal motion. The additional terms in eq. (45) are the familiar Coriolis and centrifugal accelerations associated with the steadily rotating reference frame, while the boundary and initial conditions for the problem have identical form to those in Section 2.2. Within this derivation, it has been necessary to use subscripts to denote terms associated with the internal motion $\varphi_{b}$ relative to the steadily rotating reference frame. In the remainder of this paper, however, we shall always work relative to such a steadily rotating reference frame with angular velocity $\boldsymbol{\Omega}$, and will from here on drop the associated subscripts to avoid notational clutter.

\subsection{Applications to a fluid-solid body}

We now extend Hamilton's principle to a simple hyperelastic body comprised of fluid and solid sub-bodies. For simplicity, we assume that the reference body $M$ is diffeomorphic to a closed ball in $\mathbb{R}^{3}$, and that it is decomposed in the form $M=M_{1} \cup M_{2}$ with referential fluid-solid boundary $\Sigma=M_{1} \cap M_{2}$ being diffeomorphic to a two-sphere. We let $M_{1}$ denote the enclosed 'core' of the body, but note that it does not matter which of the sub-bodies is fluid in what follows. Practically speaking, this situation corresponds to the case of a planet with a fluid core and solid mantle, or a solid planet with a global surface ocean. The modifications necessary to extend our approach to more complicated bodies (e.g. those, such as the Earth, possessing a solid inner core) are largely notational, and will be outlined in Section 2.3.3.

A simple hyperelastic material is fluid if its strain energy function $W$ depends on the deformation gradient $\mathbf{F}$ only through the Jacobian $J=\operatorname{det} \mathbf{F}$, and in this case we can write

$W(\mathbf{x}, t, \mathbf{F})=V(\mathbf{x}, t, J)$,

for some auxiliary strain energy function $V$ (e.g. Noll 1974). Physically, such a constitutive relation describes a compressible but inviscid fluid. Making use of Jacobi's formula

$\frac{\partial J}{\partial \mathbf{F}}=J \mathbf{F}^{-T}$,

where $\mathbf{F}^{-T}$ denotes the inverse transpose of the deformation gradient (e.g. Holzapfel 2000, section 1.7), the first Piola-Kirchhoff stress tensor in such a fluid takes the form

$\mathbf{T}=-p J \mathbf{F}^{-T}$, 
where $p=-\partial V / \partial J$ is the referential pressure.

Consider two particles on either side of the fluid-solid boundary that are instantaneously adjacent at some time. During the subsequent motion of the body, these particles can separate from one another as the fluid slides tangentially past the moving solid interface. It follows that the motion of such a body can be discontinuous across the fluid-solid boundary, and so we must modify the definition of the problem's configuration space. If, however, we consider the restriction $\varphi_{i}=\left.\varphi\right|_{M_{i} \times I}$ of the motion to the subregion $M_{i}$, then this should still be a smooth orientation preserving embedding into $\mathbb{R}^{3}$ for each given time. Furthermore, as the images of $M_{1}$ and $M_{2}$ cannot separate nor interpenetrate during the overall motion of the body, we require that the image of the boundary $\Sigma$ under the two mappings satisfies

$\varphi_{1}(\Sigma, t)=\varphi_{2}(\Sigma, t)$,

for $t \in I$. This relation does not require the point-wise equality $\varphi_{1}(\mathbf{x}, t)=\varphi_{2}(\mathbf{x}, t)$, but only the weaker condition that for each $\mathbf{x} \in \Sigma$ there is, at time $t$, a unique point $\mathbf{x}^{\prime} \in \Sigma$ such that $\varphi_{1}(\mathbf{x}, t)=\varphi_{2}\left(\mathbf{x}^{\prime}, t\right)$. The problem's configuration space, therefore, comprises ordered pairs

$\left(\varphi_{1}, \varphi_{2}\right) \in \operatorname{Emb}\left(M_{1} ; \mathbb{R}^{3}\right) \times \operatorname{Emb}\left(M_{2} ; \mathbb{R}^{3}\right)$

of orientation preserving embeddings subject to the tangential slip constraint in eq. (49). There is no potential energy associated specifically with such tangential slip at a fluid-solid boundary, and so the action of a fluid-solid body relative to a steadily rotating reference frame is identical to that in eq. (42). It will, however, be useful to regard this action as a function of the mappings $\varphi_{1}$ and $\varphi_{2}$ separately, and so write

$\mathcal{S}\left[\boldsymbol{\varphi}_{1}, \boldsymbol{\varphi}_{2}\right]:=\sum_{j=1}^{2} \int_{I} \int_{M_{j}}\left\{\frac{1}{2} \rho_{j}(\mathbf{x})\left\|\mathbf{v}_{j}(\mathbf{x}, t)+\boldsymbol{\Omega} \times \boldsymbol{\varphi}_{j}(\mathbf{x}, t)\right\|^{2}-W_{j}\left[\mathbf{x}, t, \mathbf{F}_{j}(\mathbf{x}, t)\right]-\frac{1}{2} \rho_{j}(\mathbf{x}) \zeta_{j}(\mathbf{x}, t)\right\} \mathrm{d}^{3} \mathbf{x} \mathrm{d} t$,

where we have added subscripts to the various terms to denote the sub-bodies in which they are defined, and where the restriction $\zeta_{j}$ of the referential gravitational potential to the sub-body $M_{j}$ can be decomposed in the form

$\zeta_{j}(\mathbf{x}, t):=\sum_{k=1}^{2} \int_{M_{k}} \rho_{k}\left(\mathbf{x}^{\prime}\right) \Gamma\left[\boldsymbol{\varphi}_{j}(\mathbf{x}, t)-\boldsymbol{\varphi}_{k}\left(\mathbf{x}^{\prime}, t\right)\right] \mathrm{d}^{3} \mathbf{x}^{\prime}$.

\subsubsection{A first derivation using constrained variations}

Starting from the action in eq. (51), we will directly apply Hamilton's principle to obtain the equations of motion for the body. In doing so, we must take account of the tangential slip constraint in eq. (49) which limits the admissible variation about the motion. All such variations of the motion

$\epsilon \mapsto\left\{\bar{\varphi}_{1}(\cdot, \cdot, \epsilon), \bar{\varphi}_{2}(\cdot, \cdot, \epsilon)\right\}$,

must satisfy eq. (49), and so the composite mapping

$\bar{\chi}(\cdot, t, \epsilon)=\left.\left.\bar{\varphi}_{1}(\cdot, t, \epsilon)\right|_{\Sigma} ^{-1} \circ \bar{\varphi}_{2}(\cdot, t, \epsilon)\right|_{\Sigma}$,

is a well-defined element of the diffeomorphism group $\operatorname{Diff}(\Sigma)$ (note here that $\circ$ denotes the composition of two functions as defined by $(f \circ g)(x)=f[g(x)]$. As the curve $\epsilon \mapsto \bar{\chi}(\mathbf{x}, t, \epsilon) \in \Sigma$ passes through the point $\chi(\mathbf{x}, t):=\bar{\chi}(\mathbf{x}, t, 0)$ when $\epsilon=0$, it follows that the vector

$\delta \chi(\mathbf{x}, t)=\left(\partial_{\epsilon} \chi\right)(\mathbf{x}, t, 0)$

lies in the tangent space of $\Sigma$ at $\chi(\mathbf{x}, t)$. For any $\mathbf{x} \in \Sigma$, we have by definition

$\bar{\varphi}_{1} \circ \bar{\chi}=\bar{\varphi}_{2}$,

and differentiating with respect to $\epsilon$, we obtain

$\delta \boldsymbol{\varphi}_{1} \circ \chi+\left(\mathbf{F}_{1} \circ \chi\right) \cdot \delta \chi=\delta \boldsymbol{\varphi}_{2}$,

where we have defined

$\delta \boldsymbol{\varphi}_{j}=\left.\frac{\partial \overline{\boldsymbol{\varphi}}_{j}}{\partial \epsilon}\right|_{\epsilon=0}$.

Using this result, we can obtain the equations of motion by setting

$\delta \mathcal{S}=\left.\frac{\mathrm{d}}{\mathrm{d} \epsilon} \mathcal{S}\left[\overline{\boldsymbol{\varphi}}_{1}(\cdot, \cdot, \epsilon), \bar{\varphi}_{2}(\cdot, \cdot, \epsilon)\right]\right|_{\epsilon=0}=0$, 
for all possible variations of the motion. Calculation of the first variation of the action proceeds almost exactly as in section 2.5 of AC16, and leads to

$$
\begin{aligned}
\delta \mathcal{S}= & -\sum_{j=1}^{2} \int_{I} \int_{M_{j}}\left\langle\rho \partial_{t} \mathbf{v}_{j}+2 \rho_{j} \boldsymbol{\Omega} \times \mathbf{v}_{j}+\rho_{j} \boldsymbol{\Omega} \times\left(\boldsymbol{\Omega} \times \boldsymbol{\varphi}_{j}\right)-\operatorname{Div}_{j}-\rho_{j} \boldsymbol{\gamma}_{j}, \delta \boldsymbol{\varphi}_{j}\right\rangle \mathrm{d}^{3} \mathbf{x} \mathrm{d} t \\
& -\int_{I} \int_{\partial M}\left\langle\mathbf{t}_{2}, \delta \boldsymbol{\varphi}_{2}\right\rangle \mathrm{d} S \mathrm{~d} t+\int_{I} \int_{\Sigma}\left\langle\mathbf{t}_{2}, \delta \boldsymbol{\varphi}_{2}\right\rangle \mathrm{d} S \mathrm{~d} t-\int_{I} \int_{\Sigma}\left\langle\mathbf{t}_{1}, \delta \boldsymbol{\varphi}_{1}\right\rangle \mathrm{d} S \mathrm{~d} t,
\end{aligned}
$$

where $\mathbf{T}_{j}$ is the first Piola-Kirchhoff stress tensor within the $j$ th sub-body, $\mathbf{t}_{j}=\mathbf{T}_{j} \cdot \hat{\mathbf{n}}$ is the associated referential traction vector, and $\boldsymbol{\gamma}_{j}$ is the restriction of the referential gravitational acceleration to $M_{j}$ which can be decomposed as

$\boldsymbol{\gamma}_{j}(\mathbf{x}, t):=-\sum_{k=1}^{2} \int_{M_{k}} \rho_{j}\left(\mathbf{x}^{\prime}\right)(\nabla \Gamma)\left[\boldsymbol{\varphi}_{j}(\mathbf{x}, t)-\boldsymbol{\varphi}_{k}\left(\mathbf{x}^{\prime}, t\right)\right] \mathrm{d}^{3} \mathbf{x}^{\prime}$.

As $\delta \varphi_{j}$ within the interior of $M_{j}$ is arbitrary, we obtain the usual momentum equation

$\rho_{j} \partial_{t} \mathbf{v}_{j}+2 \rho_{j} \boldsymbol{\Omega} \times \mathbf{v}_{j}+\rho_{j} \boldsymbol{\Omega} \times\left(\boldsymbol{\Omega} \times \boldsymbol{\varphi}_{j}\right)-\operatorname{Div}_{j}-\rho_{j} \boldsymbol{\gamma}_{j}=\mathbf{0}$,

within each sub-body, and similarly arrive at the free-surface condition $\mathbf{t}_{2}=\mathbf{0}$ on $\partial M$. To deal with the remaining boundary terms, we recall that the variations of the motion are subject to eq. (57). We then use $\chi(\cdot, t) \in \operatorname{Diff}(\Sigma)$ to perform the following change of variables:

$\int_{\Sigma}\left\langle\mathbf{t}_{1}, \delta \varphi_{1}\right\rangle \mathrm{d} S=\int_{\Sigma} J_{\chi}\left\langle\mathbf{t}_{1} \circ \chi, \delta \varphi_{1} \circ \chi\right\rangle \mathrm{d} S$,

where $J_{\chi}$ is the surface Jacobian associated with $\chi(\cdot, t) \in \operatorname{Diff}(\Sigma)$ (e.g. Abraham et al. 2012), and using eq. (57) to relate the perturbations $\delta \varphi_{1}$ and $\delta \varphi_{2}$ on $\Sigma$, we arrive at

$\int_{I} \int_{\Sigma}\left\langle\mathbf{t}_{2}, \delta \boldsymbol{\varphi}_{2}\right\rangle \mathrm{d} S \mathrm{~d} t-\int_{I} \int_{\Sigma}\left\langle\mathbf{t}_{1}, \delta \boldsymbol{\varphi}_{1}\right\rangle \mathrm{d} S \mathrm{~d} t=\int_{I} \int_{\Sigma}\left\langle\mathbf{t}_{2}-J_{\chi} \mathbf{t}_{1} \circ \chi, \delta \boldsymbol{\varphi}_{2}\right\rangle \mathrm{d} S \mathrm{~d} t+\int_{I} \int_{\Sigma} J_{\chi}\left\langle\left(\mathbf{F}_{1} \circ \chi\right)^{T} \cdot\left(\mathbf{t}_{1} \circ \chi\right), \delta \chi\right\rangle \mathrm{d} S \mathrm{~d} t$.

The perturbation $\delta \varphi_{2}$ is arbitrary on $\Sigma$, and so we obtain the condition

$\mathbf{t}_{2}=J_{\chi} \mathbf{t}_{1} \circ \chi$

The perturbation $\delta \chi$ is subject to the constraint that it is tangent to $\Sigma$ at $\chi(\mathbf{x}, t)$, and this implies that $\left(\mathbf{F}_{1} \circ \chi\right)^{T} \cdot\left(\mathbf{t}_{1} \circ \chi\right)$ must be normal to $\Sigma$ at this point. Recalling the form of the first Piola-Kirchhoff stress tensor within a fluid given in eq. (48), it is then clear that

$\mathbf{t}_{1}=-p_{1} J_{1} \mathbf{F}_{1}^{-T} \cdot \hat{\mathbf{n}}$.

The first condition in eq. (65) expresses the continuity of traction between surface elements located on the lower and upper sides of the fluidsolid boundary that are instantaneously adjacent - the presence of the surface Jacobian is due to these referential tractions being measured per unit area at different points on the reference boundary. The second condition in eq. (66) shows that the traction on the fluid--solid boundary is, as expected, always parallel to the boundary's instantaneous normal. These two conditions at a fluid-solid boundary are equivalent to those obtained in the original discussion of this problem by Woodhouse \& Dahlen (1978) based on Newton's third law of motion. As noted in Section 1, Brazda et al. (2017) have also recently presented a variational principle for such fluid-solid elastic bodies. While the final form of the equations of motion they obtain is equivalent to ours, it is notable that these authors include an additional surface integral term into their action associated with tangential slip. The physical meaning and need for such a term is, in our view, unclear.

\subsubsection{A second derivation using Lagrange multipliers}

We now show that the equations of motion for a fluid-solid body can also be obtained from an unconstrained variational principle through the introduction of an appropriate Lagrangian multiplier field. When considering the linearized equations of motion, it will be this second variational principle that is easiest to apply, as we need not then consider the tangential slip constraint explicitly when forming a quadratic expansion of the action.

To proceed, we must reformulate the tangential slip constraint given in eq. (49) in a point-wise manner. As we assumed that $\Sigma$ is diffeomorphic to a two-sphere, there exists a smooth function $\sigma: \mathbb{R}^{3} \rightarrow \mathbb{R}$ such that this surface can be expressed in the form

$\Sigma=\sigma^{-1}(0):=\left\{\mathbf{x} \in \mathbb{R}^{3} \mid \sigma(\mathbf{x})=0\right\}$,

and where $\left.\nabla \sigma\right|_{\Sigma}$ is everywhere non-zero (e.g. Abraham et al. 2012). For example, if $\Sigma$ is equal to the unit two-sphere, then we can take $\sigma(\mathbf{x})=\|\mathbf{x}\|-1$. Note that $\left.\nabla \sigma\right|_{\Sigma}$ is parallel to the boundary's outward unit normal vector $\hat{\mathbf{n}}$, and that $\sigma$ can always be chosen so that these two vector fields are equal if desired. Given such a function, then for any element $\chi$ of the diffeomorphism group Diff( $\Sigma)$ we have $\sigma \circ \chi=0$ identically. If a pair of embeddings $\left(\boldsymbol{\varphi}_{1}, \boldsymbol{\varphi}_{2}\right) \in \operatorname{Emb}\left(M_{1} ; \mathbb{R}^{3}\right) \times \operatorname{Emb}\left(M_{2} ; \mathbb{R}^{3}\right)$ do satisfy eq. (49), then we have noted previously that $\varphi_{1}^{-1} \circ \varphi_{2} \in \operatorname{Diff}(\Sigma)$, and so we obtain

$\sigma \circ \varphi_{1}^{-1} \circ \varphi_{2}=0$ 
on all of $\Sigma$. This condition is almost the required point-wise expression of the tangential slip constraint, but it is not well defined for all elements of $\operatorname{Emb}\left(M_{1} ; \mathbb{R}^{3}\right) \times \operatorname{Emb}\left(M_{2} ; \mathbb{R}^{3}\right)$. Indeed, the composition $\left.\left(\varphi_{1}^{-1} \circ \varphi_{2}\right)\right|_{\Sigma}$ can only be formed if we have $\varphi_{2}(\Sigma) \subseteq \varphi_{1}\left(M_{1}\right)$. To remove this problem, we now require that $\varphi_{1}$ belongs to the diffeomorphism group Diff $\left(\mathbb{R}^{3}\right)$, and hence that $\varphi_{1}^{-1}$ is well defined for all points in $\mathbb{R}^{3}$. Having done this, it is clear that if $\left(\boldsymbol{\varphi}_{1}, \boldsymbol{\varphi}_{2}\right) \in \operatorname{Diff}\left(\mathbb{R}^{3}\right) \times \operatorname{Emb}\left(M_{2} ; \mathbb{R}^{3}\right)$ is such that eq. (68) holds, then $\left(\left.\boldsymbol{\varphi}_{1}\right|_{M_{1}}, \boldsymbol{\varphi}_{2}\right) \in \operatorname{Emb}\left(M_{1} ; \mathbb{R}^{3}\right) \times \operatorname{Emb}\left(M_{2} ; \mathbb{R}^{3}\right)$ satisfies the tangential slip condition as previously formulated.

Having obtained this point-wise formulation of the tangential slip constraint, we introduce the following modified action for the fluid-solid body

$$
\begin{aligned}
\mathcal{S}\left[\boldsymbol{\varphi}_{1}, \boldsymbol{\varphi}_{2}, \varpi\right]:= & \sum_{j=1}^{2} \int_{I} \int_{M_{j}}\left\{\frac{1}{2} \rho_{j}(\mathbf{x})\left\|\mathbf{v}_{j}(\mathbf{x}, t)+\boldsymbol{\Omega} \times \boldsymbol{\varphi}_{j}(\mathbf{x}, t)\right\|^{2}-W_{j}\left[\mathbf{x}, t, \mathbf{F}_{j}(\mathbf{x}, t)\right]-\frac{1}{2} \rho_{j}(\mathbf{x}) \zeta_{j}(\mathbf{x}, t)\right\} \mathrm{d}^{3} \mathbf{x} \mathrm{d} t \\
& +\int_{I} \int_{\Sigma} \varpi(\mathbf{x}, t)(\sigma \circ \chi)(\mathbf{x}, t) \mathrm{d} S \mathrm{~d} t,
\end{aligned}
$$

where for each time $\left(\varphi_{1}(\cdot, t), \varphi_{2}(\cdot, t)\right) \in \operatorname{Diff}\left(\mathbb{R}^{3}\right) \times \operatorname{Emb}\left(M_{2} ; \mathbb{R}^{3}\right)$, and we have defined

$\chi(\cdot, t)=\left.\varphi_{1}(\cdot, t)^{-1} \circ \varphi_{2}(\cdot, t)\right|_{\Sigma}$,

for convenience. This action is a function of the motions $\varphi_{1}$ and $\varphi_{2}$ along with the Lagrange multiplier field $\varpi$ needed to enforce the tangential slip constraint in eq. (68). Importantly, within the action, the fields $\varphi_{1}, \varphi_{2}$ and $\varpi$ are all subject to independent variations. It will clear below that the form of $\varphi_{1}$ outside of $M_{1}$ is not determined by, and has no effect on, the resulting equations of motion. The extension of this mapping to be defined on all of $\mathbb{R}^{3}$ is, however, a necessary technical artifice that allows the tangential slip constraint in eq. (49) to be suitably reformulated within the framework of Lagrangian multipliers.

To obtain the equations of motion, we again need to calculate the first variation of the action with respect to each of its arguments. The variation with respect to the Lagrangian multiplier field is trivial, and yields the tangential slip condition in eq. (68) which we assume to hold for the remainder of the derivation. Variation of the volumetric terms in the action leads, as before, to eq. (60). To vary the surface integral term with respect to the motion, we first use the fact that $\chi(\cdot, t) \in \operatorname{Diff}(\Sigma)$ to write

$\varphi_{1} \circ \chi=\varphi_{2}$,

where it is understood that the composition is with respect to the spatial variables at a fixed time. Using the chain rule, we obtain the relation

$\delta \chi=\left(\mathbf{F}_{1} \circ \chi\right)^{-1} \cdot\left(\delta \varphi_{2}-\delta \varphi_{1} \circ \chi\right)$,

which is equivalent to eq. (57). The first variation of the action can now be written

$$
\begin{aligned}
\delta \mathcal{S}= & -\sum_{j=1}^{2} \int_{I} \int_{M_{j}}\left\langle\rho \partial_{t} \mathbf{v}_{j}+2 \rho_{j} \boldsymbol{\Omega} \times \mathbf{v}_{j}+\rho_{j} \boldsymbol{\Omega} \times\left(\boldsymbol{\Omega} \times \boldsymbol{\varphi}_{j}\right)-\operatorname{Div} \mathbf{T}_{j}-\rho_{j} \boldsymbol{\gamma}_{j}, \delta \boldsymbol{\varphi}_{j}\right\rangle \mathrm{d}^{3} \mathbf{x} \mathrm{d} t-\int_{I} \int_{\partial M}\left\langle\mathbf{t}_{2}, \delta \boldsymbol{\varphi}_{2}\right\rangle \mathrm{d} S \mathrm{~d} t \\
& +\int_{I} \int_{\Sigma}\left\langle\mathbf{t}_{2}, \delta \boldsymbol{\varphi}_{2}\right\rangle \mathrm{d} S \mathrm{~d} t-\int_{I} \int_{\Sigma}\left\langle\mathbf{t}_{1}, \delta \boldsymbol{\varphi}_{1}\right\rangle \mathrm{d} S \mathrm{~d} t+\int_{I} \int_{\Sigma} \varpi\left\langle\left(\mathbf{F}_{1} \circ \chi\right)^{-T} \cdot(\nabla \sigma \circ \boldsymbol{\chi}), \delta \boldsymbol{\varphi}_{2}-\delta \boldsymbol{\varphi}_{1} \circ \chi\right\rangle \mathrm{d} S \mathrm{~d} t,
\end{aligned}
$$

and using use $\chi(\cdot, t)$ to change variables within the fourth term, we obtain

$$
\begin{aligned}
\delta \mathcal{S}= & -\sum_{j=1}^{2} \int_{I} \int_{M_{j}}\left\langle\rho_{j} \partial_{t} \mathbf{v}_{j}+2 \rho_{j} \boldsymbol{\Omega} \times \mathbf{v}_{j}+\rho_{j} \boldsymbol{\Omega} \times\left(\boldsymbol{\Omega} \times \boldsymbol{\varphi}_{j}\right)-\operatorname{Div}_{j}-\rho_{j} \boldsymbol{\gamma}_{j}, \delta \boldsymbol{\varphi}_{j}\right\rangle \mathrm{d}^{3} \mathbf{x} \mathrm{d} t-\int_{I} \int_{\partial M}\left\langle\mathbf{t}_{2}, \delta \boldsymbol{\varphi}_{2}\right\rangle \mathrm{d} S \mathrm{~d} t \\
& +\int_{I} \int_{\Sigma}\left\langle\mathbf{t}_{2}+\varpi\left(\mathbf{F}_{1} \circ \chi\right)^{-T} \cdot(\nabla \sigma \circ \chi), \delta \boldsymbol{\varphi}_{2}\right\rangle \mathrm{d} S \mathrm{~d} t-\int_{I} \int_{\Sigma}\left\langle J_{\chi} \mathbf{t}_{1} \circ \chi+\varpi\left(\mathbf{F}_{1} \circ \chi\right)^{-T} \cdot(\nabla \sigma \circ \chi), \delta \boldsymbol{\varphi}_{1} \circ \chi\right\rangle \mathrm{d} S \mathrm{~d} t .
\end{aligned}
$$

The first variation of the action is required to vanish for arbitrary $\delta \varphi_{1}$ and $\delta \varphi_{2}$, and so we again arrive at the usual momentum equation

$\rho_{j} \partial_{t} \mathbf{v}_{j}+2 \rho_{j} \boldsymbol{\Omega} \times \mathbf{v}_{j}+\rho_{j} \boldsymbol{\Omega} \times\left(\boldsymbol{\Omega} \times \boldsymbol{\varphi}_{j}\right)-\operatorname{Div}_{j}-\rho_{j} \boldsymbol{\gamma}_{j}=\mathbf{0}$,

within each sub-body $M_{j}$. From the boundary terms we also immediately obtain the expected traction-free boundary condition $\mathbf{t}_{2}=\mathbf{0}$ on $\partial M$, along with the following two conditions on

$\mathbf{t}_{2}+\varpi\left(\mathbf{F}_{1} \circ \chi\right)^{-T} \cdot(\nabla \sigma \circ \chi)=\mathbf{0}, \quad J_{\chi} \mathbf{t}_{1} \circ \chi+\varpi\left(\mathbf{F}_{1} \circ \chi\right)^{-T} \cdot(\nabla \sigma \circ \chi)=\mathbf{0}$,

on $\Sigma$, which are readily seen to imply

$\mathbf{t}_{2}=J_{\chi} \mathbf{t}_{1} \circ \chi=-\varpi\left(\mathbf{F}_{1} \circ \chi\right)^{-T} \cdot(\nabla \sigma \circ \chi)$.

The first of these equalities is just a restatement of eq. (65), while the second is equivalent to eq. (66) because $\left.\nabla \sigma\right|_{\Sigma}$ is, by construction, parallel to $\hat{\mathbf{n}}$. Furthermore, from eq. (76) we see that the Lagrange multiplier field $\varpi$ is given by

$\varpi=-\frac{\left\langle\left(\mathbf{F}_{1} \circ \chi\right)^{-T} \cdot(\nabla \sigma \circ \chi), \mathbf{t}_{2}\right\rangle}{\left\|\left(\mathbf{F}_{1} \circ \chi\right)^{-T} \cdot(\nabla \sigma \circ \chi)\right\|^{2}}$, 
and is, therefore, proportional to the pressure on the fluid-solid boundary. In this manner, we have shown that these two variational principles in a fluid-solid body are indeed equivalent.

\subsubsection{Extension of the method to more complex bodies}

The preceding derivations placed fairly strong restrictions on the topology of the body. These methods can, however, be extended to more general bodies at the expense of more elaborate notations to label the different sub-bodies and their mutual boundaries. In particular, for any smooth, closed and orientated 2-D surface there exists functions $\sigma: \mathbb{R}^{3} \rightarrow \mathbb{R}$ with the properties required for the point-wise formulation of the tangential slip constraint in eq. (68). The only real limitation of our approach is that it does not permit intersecting boundaries between solid and fluid subregions. The most obvious practical example of such intersecting boundaries is the shoreline associated with a non-global ocean. If, in such a model, the motion was small enough that the topology of the ocean basins remains unchanged, then our present methods could be suitably adapted. If, however, the topology of the ocean basins is changed - through, for example, the coalescence of two initially separate water bodies during the motion - then the problem could never be adequately described using a fixed reference body. This issue is, however, unlikely to be important in solid-earth geophysics, and will not be considered further in the present paper. Indeed, for many applications at a global-scale it is sufficient to treat the oceans as effective surface loads (e.g. Dahlen 1976; Farrell \& Clark 1976; Komatitsch \& Tromp 2002).

\subsection{Particle relabelling transformations}

Within the discussion so far, a fluid-solid simple hyperelastic body has been specified in terms of a reference body $M=M_{1} \cup M_{2} \subseteq \mathbb{R}^{3}$ subject to the stated topological restrictions, along with material parameters $\left(\rho_{j}, W_{j}\right)$ defined on $M_{j}$ for $j=1,2$. Such a description is not unique, but depends on the choice of reference configuration. As discussed in AC16 and in Section 1, for any orientation preserving diffeomorphism $\xi: \tilde{M} \rightarrow M$ there is a corresponding particle relabelling transformation, and this can be used to define a new reference configuration for the body which has material parameters $\left(\tilde{\rho}_{j}, \tilde{W}_{j}\right)$. The motion of the body $\tilde{\varphi}_{j}: \tilde{M}_{j} \times \mathbb{R} \rightarrow \mathbb{R}^{3}$ for $j=1,2$ relative to the new reference configuration is defined through the identity

$\tilde{\boldsymbol{\varphi}}_{j}(\mathbf{x}, t)=\varphi_{j}[\boldsymbol{\xi}(\mathbf{x}), t]$,

for all $(\mathbf{x}, t) \in \tilde{M} \times \mathbb{R}$. The referential fluid-solid boundary in $\tilde{M}$ is given by $\tilde{\Sigma}=\xi^{-1}(\Sigma)$, and we impose the following transformation rule on the Lagrangian multiplier fields

$\tilde{\varpi}(\mathbf{x}, t)=\left.J_{\xi}\right|_{\tilde{\Sigma}}(\mathbf{x}) \varpi[\xi(\mathbf{x}), t]$,

where $\left.J_{\xi}\right|_{\tilde{\Sigma}}$ is the surface Jacobian corresponding to the restricted mapping $\left.\xi\right|_{\tilde{\Sigma}}: \tilde{\Sigma} \rightarrow \Sigma$. This expression is motivated by the fact that $\varpi$ is proportional to pressure on the fluid-solid boundary, and so would be expected to behave like a differential two-form on a surface (e.g. Abraham et al. 2012). With respect to the new reference configuration, the motion of the body is governed by the action

$$
\begin{aligned}
\tilde{\mathcal{S}}\left[\tilde{\boldsymbol{\varphi}}_{1}, \tilde{\boldsymbol{\varphi}}_{2}, \tilde{\varpi}\right]:= & \sum_{j=1}^{2} \int_{I} \int_{\tilde{M}_{j}}\left\{\frac{1}{2} \tilde{\rho}_{j}(\mathbf{x})\left\|\tilde{\mathbf{v}}_{j}(\mathbf{x}, t)+\mathbf{\Omega} \times \tilde{\boldsymbol{\varphi}}_{j}(\mathbf{x}, t)\right\|^{2}-\tilde{W}_{j}\left[\mathbf{x}, t, \tilde{\mathbf{F}}_{j}(\mathbf{x}, t)\right]-\frac{1}{2} \tilde{\rho}_{j}(\mathbf{x}) \tilde{\zeta}_{j}(\mathbf{x}, t)\right\} \mathrm{d}^{3} \mathbf{x} \mathrm{d} t \\
& +\int_{I} \int_{\tilde{\Sigma}} \tilde{\varpi}(\mathbf{x}, t)(\tilde{\sigma} \circ \tilde{\chi})(\mathbf{x}, t) \mathrm{d} S \mathrm{~d} t,
\end{aligned}
$$

having identical form to eq. (69). In order for the actions in eqs (69) and (81) to describe to the same physical process, we require

$\mathcal{S}\left[\boldsymbol{\varphi}_{1}, \boldsymbol{\varphi}_{2}, \varpi\right]=\tilde{\mathcal{S}}\left[\tilde{\varphi}_{1}, \tilde{\varphi}_{2}, \tilde{\varpi}\right]$,

whenever the arguments on either side are related through the above transformations. Using $\xi: \tilde{M} \rightarrow M$ to change variables in eq. (69), it is then easy to see that eq. (82) will hold if the material parameters in the two descriptions are related by

$\tilde{\rho}_{j}(\mathbf{x})=J_{\xi}(\mathbf{x}) \rho_{j}[\boldsymbol{\xi}(\mathbf{x}), t], \quad \tilde{W}_{j}(\mathbf{x}, t, \tilde{\mathbf{F}})=J_{\xi}(\mathbf{x}) W_{j}\left[\boldsymbol{\xi}(\mathbf{x}), t, \tilde{\mathbf{F}} \mathbf{F}_{\xi}^{-1}\right]$,

where $\mathbf{F}_{\xi}=\operatorname{Def} \xi$ and $J_{\xi}=\operatorname{det} \mathbf{F}_{\xi}$. These transformations are unique up to the addition of a term independent of $\mathbf{F}$ to the strain energy function $\tilde{W}$, but this would have no physical significance. These results are identical to those obtained within AC16, and show that particle relabelling transformations extend to fluid-solid bodies in a simple manner.

\section{EQUILIBRIUM CONFIGURATIONS AND LINEARIZED EQUATIONS OF MOTION}

\subsection{Perturbation series expansion of the action}

In this section, we outline a standard approach for obtaining the linearized equations of motion about an equilibrium configuration for a system governed by Hamilton's principle. This method, in particular, leads directly to a variational principle for the linearized equations of 
motion, and greatly simplifies the derivation of the weak formulation of the problem needed within numerical calculations. Recalling the decomposition of the body's strain energy function in eq. (84) into a constitutive part and a stress-glut term, we now write

$W(\mathbf{x}, t, \mathbf{F})=U(\mathbf{x}, \mathbf{C})-\frac{1}{2} \epsilon\langle\mathfrak{S}(\mathbf{x}, t), \mathbf{C}\rangle$,

where $\epsilon$ is a dimensionless perturbation parameter. When $\epsilon=0$, no stress glut acts on the body, and we assume that its motion is described by a time-independent equilibrium configuration $\left\{\varphi_{1}^{(0)}, \varphi_{2}^{(0)}\right\}$. For non-zero values of $\epsilon$, the body will be disturbed from this equilibrium, and we assume that the resulting motion can be expanded in the perturbation series

$\boldsymbol{\varphi}_{j}(\mathbf{x}, t)=\boldsymbol{\varphi}_{j}^{(0)}(\mathbf{x})+\sum_{m=1}^{\infty} \epsilon^{m} \boldsymbol{\varphi}_{j}^{(m)}(\mathbf{x}, t)$,

and similarly for the Lagrange multiplier field

$\varpi_{j}(\mathbf{x}, t)=\varpi_{j}^{(0)}(\mathbf{x})+\sum_{m=1}^{\infty} \epsilon^{m} \varpi_{j}^{(m)}(\mathbf{x}, t)$.

As there seems no practical reason to do otherwise, we require that the equilibrium motion be continuous across $\Sigma$, meaning that we have

$\left.\varphi_{1}^{(0)}\right|_{\Sigma}=\left.\varphi_{2}^{(0)}\right|_{\Sigma}$

Substituting these expressions into the action in eq. (69), we arrive at a perturbation series expansion of the action in powers of $\epsilon$ taking the following form:

$\mathcal{S}=\sum_{m=0}^{\infty} \epsilon^{m} \mathcal{S}^{(m)}$.

In order for Hamilton's principle to hold, it is necessary for each of the resulting actions $\mathcal{S}^{(m)}$ to be stationary with respect to arbitrary variations of its arguments. We note, however, that all such variations are subject to fixed initial and terminal conditions, and so the equilibrium values $\varphi_{1}^{(0)}, \varphi_{2}^{(0)}$ and $\varpi^{(0)}$ are not themselves varied. From variation of the first-order terms we obtain the equilibrium equations, while variation of the second-order terms leads to the linearized equations of motion. Note that there is a linear contribution to the second-order action from the second-order fields $\left(\varphi_{1}^{(2)}, \varphi_{2}^{(2)}, \varpi^{(2)}\right)$, but variation of these terms just gives the equilibrium equations again. Continuing in this manner, we could, if desired, obtain linear equations satisfied by the higher-order terms in the expansion of the motion, and so account progressively for nonlinearity in the problem.

\subsection{Equilibrium configurations}

From eq. (69) and the calculations within Section 2.3.2, we find that the first-order contribution to the perturbation series in eq. (88) is given by

$$
\begin{aligned}
\mathcal{S}^{(1)}= & -\sum_{j=1}^{2} \int_{I} \int_{M_{j}}\left\langle\rho_{j} \boldsymbol{\Omega} \times\left(\boldsymbol{\Omega} \times \boldsymbol{\varphi}_{j}^{(0)}\right)-\operatorname{Div} \mathbf{T}_{j}^{(0)}-\rho_{j} \boldsymbol{\gamma}_{j}^{(0)}, \delta \boldsymbol{\varphi}_{j}^{(1)}\right\rangle \mathrm{d}^{3} \mathbf{x} \mathrm{d} t-\int_{I} \int_{\partial M}\left\langle\mathbf{t}_{2}^{(0)}, \delta \boldsymbol{\varphi}_{2}^{(1)}\right\rangle \mathrm{d} S \mathrm{~d} t \\
& +\int_{I} \int_{\Sigma}\left\langle\mathbf{t}_{2}^{(0)}+\varpi^{(0)} \mathbf{F}_{1}^{(0)-T} \cdot \nabla \sigma, \delta \boldsymbol{\varphi}_{2}^{(1)}\right\rangle \mathrm{d} S \mathrm{~d} t-\int_{I} \int_{\Sigma}\left\langle\mathbf{t}_{1}^{(0)}+\varpi^{(0)} \mathbf{F}_{1}^{(0)-T} \cdot \nabla \sigma, \delta \boldsymbol{\varphi}_{1}^{(1)}\right\rangle \mathrm{d} S \mathrm{~d} t,
\end{aligned}
$$

where superscripts have been added to terms associated with the equilibrium motions in an obvious manner. Note that in obtaining this result, we made use eq. (92) to conclude that $\chi$ defined in eq. (70) is equal to identity the mapping to zeroth order in $\epsilon$. Requiring this action vanish for arbitrary variations, we arrive at the equilibrium equation

$\rho_{j} \boldsymbol{\Omega} \times\left(\boldsymbol{\Omega} \times \boldsymbol{\varphi}_{j}^{(0)}\right)-\operatorname{DivT}_{j}^{(0)}-\rho_{j} \boldsymbol{\gamma}_{j}^{(0)}=\mathbf{0}$,

along with the traction-free boundary condition

$\mathbf{t}_{2}^{(0)}=\mathbf{0}$,

on $\partial M$, and the continuity conditions

$\mathbf{t}_{1}^{(0)}=\mathbf{t}_{2}^{(0)}=-\varpi^{(0)} \mathbf{F}_{1}^{(0)-T} \cdot \nabla \sigma$,

on $\Sigma$. These results could, of course, have been obtained directly from the exact equations of motion and boundary conditions in Section 2.3.2, but it is interesting to see them arise in a natural manner from the perturbation expansion of the action.

We shall not consider equilibrium configurations in detail, but several points are worth noting. First, for a given strain energy function, these equilibrium equations represent a system of nonlinear integro-partial differential equations that could, in principle, be solved to determine an equilibrium configuration for the body. For geophysical applications, however, the solution of this problem has little relevance because the Earth or other similar planets are not elastic over the timescales that determine their equilibrium states. Nonetheless, the equilibrium equations 
in a more general material take exactly the same form, but with the stress tensor now being related to the motion in a complicated and usually unknown manner. We should, therefore, regard these equations as placing constraints on the referential density, the equilibrium configuration and the equilibrium stress field (e.g. Backus 1967; Al-Attar \& Woodhouse 2010). In particular, within fluid regions the equilibrium stress tensor must take the form given by eq. (48). Recalling the so-called Piola identity $\operatorname{Div}\left(J \mathbf{F}^{-T}\right)=\mathbf{0}$ (e.g. Marsden \& Hughes 1994 , theorem 7.20) which holds for the deformation gradient of any motion, it follows that within fluid regions we have

$\operatorname{Div} \mathbf{T}_{j}^{(0)}=-J_{j}^{(0)} \mathbf{F}_{j}^{(0)-T} \cdot \nabla p_{j}^{(0)}$,

where $p_{j}^{(0)}$ is the equilibrium value of the referential pressure. Through simple calculations using the chain rule, we can also write

$\boldsymbol{\gamma}_{j}^{(0)}=-\mathbf{F}_{j}^{(0)-T} \cdot \nabla \zeta_{j}^{(0)}, \quad \boldsymbol{\Omega} \times\left(\boldsymbol{\Omega} \times \boldsymbol{\varphi}_{j}^{(0)}\right)=\mathbf{F}_{j}^{(0)-T} \cdot \nabla \psi_{j}^{(0)}$,

where we have introduced the centrifugal potential

$\psi_{j}^{(0)}=\frac{1}{2}\left[\left\langle\boldsymbol{\Omega}, \boldsymbol{\varphi}_{j}^{(0)}\right\rangle^{2}-\|\boldsymbol{\Omega}\|^{2}\left\|\boldsymbol{\varphi}_{j}^{(0)}\right\|^{2}\right]$.

Within fluid regions of the body we can, therefore, write the equilibrium equations in the simpler form

$J_{j}^{(0)} \nabla p_{j}^{(0)}+\rho \nabla\left(\zeta_{j}^{(0)}+\psi_{j}^{(0)}\right)=\mathbf{0}$.

This equation closely resembles, and is equivalent to, the usual expression of hydrostatic equilibrium within a rotating and self-gravitating fluid (e.g. Jeffreys 1976; Dahlen \& Tromp 1998), but here we are working entirely within a referential description. We note that Al-Attar (2011) previously obtained this referential formulation of the equilibrium equations using a different method, and showed that this approach can be used to develop an exact, nonlinear, generalization of Clairaut's theory of rotational flattening. The practical implementation of these ideas will be discussed in future work.

\subsection{Linearized motions about equilibrium}

\subsubsection{Derivation of the quadratic action}

Turning to the linearized equations of motion, we first split the action $\mathcal{S}$ in eq. (69) into a volumetric part $\mathcal{S}_{V}$ and a surface part $\mathcal{S}_{\Sigma}$ associated with the referential fluid-solid boundary. It is a simple matter to show that the contribution to the quadratic term $\mathcal{S}^{(2)}$ in eq. (88) from $\mathcal{S}_{V}$ is given by

$\mathcal{S}_{V}^{(2)}=\sum_{j=1}^{2} \int_{I} \int_{M_{j}}\left\{\frac{1}{2} \rho_{j}\left\|\mathbf{v}_{j}^{(1)}+\boldsymbol{\Omega} \times \boldsymbol{\varphi}_{j}^{(1)}\right\|^{2}-\frac{1}{2}\left\langle\boldsymbol{\Lambda}_{j} \cdot \mathbf{F}_{j}^{(1)}-\mathfrak{S}_{j}, \mathbf{F}_{j}^{(1)}\right\rangle+\frac{1}{2} \rho_{j}\left\langle\boldsymbol{\gamma}_{j}^{(1)}, \boldsymbol{\varphi}_{j}^{(1)}\right\rangle\right\} \mathrm{d}^{3} \mathbf{x} \mathrm{d} t$,

where superscripts have been added in an obvious manner. The elastic tensor is defined by

$\mathbf{\Lambda}_{j}(\mathbf{x})=\frac{\partial^{2} W_{j}}{\partial \mathbf{F}_{j} \mathbf{F}_{j}}\left[\mathbf{x}, t, \mathbf{F}_{j}^{(0)}(\mathbf{x})\right]$

and the linearized referential gravitational acceleration can be written

$\boldsymbol{\gamma}_{j}^{(1)}(\mathbf{x})=-\sum_{k=1}^{2} \int_{M_{k}} \rho_{k}\left(\mathbf{x}^{\prime}\right)(\nabla \nabla \Gamma)\left[\boldsymbol{\varphi}_{j}^{(0)}(\mathbf{x})-\boldsymbol{\varphi}_{k}^{(0)}\left(\mathbf{x}^{\prime}\right)\right] \cdot\left[\boldsymbol{\varphi}_{j}^{(1)}(\mathbf{x})-\boldsymbol{\varphi}_{k}^{(1)}\left(\mathbf{x}^{\prime}\right)\right] \mathrm{d}^{3} \mathbf{x}^{\prime}$

Determining the contribution of the surface term $\mathcal{S}_{\Sigma}$ to the quadratic action requires more effort, as we need to expand the mapping $\chi$ defined in eq. (70) up to second order. To do this, we write

$\chi(\mathbf{x}, t)=\mathbf{x}+\sum_{m=1}^{\infty} \epsilon^{m} \chi^{(m)}(\mathbf{x}, t)$,

for $\mathbf{x} \in \Sigma$, where we have again made use of eq. (92) in determining the zeroth-order term. Substituting this expansion into the identity

$\varphi_{1} \circ \chi=\varphi_{2}$,

and using eq. (85), we find that

$\mathbf{F}_{1}^{(0)} \cdot \chi^{(1)}=\varphi_{2}^{(1)}-\varphi_{1}^{(1)}$,

$\mathbf{F}_{1}^{(0)} \cdot \chi^{(2)}=-\frac{1}{2} \mathbf{H}_{1}^{(0)} \cdot\left\{\mathbf{F}_{1}^{(0)-1}\left[\left(\varphi_{2}^{(1)}-\varphi_{1}^{(1)}\right) \otimes\left(\varphi_{2}^{(1)}-\varphi_{1}^{(1)}\right)\right] \mathbf{F}_{1}^{(0)-T}\right\}-\left(\mathbf{F}_{1}^{(1)} \mathbf{F}_{1}^{(0)-1}\right) \cdot\left(\varphi_{2}^{(1)}-\varphi_{1}^{(1)}\right)$,

where $\otimes$ denotes the tensor product, and we have introduced

$\left[\mathbf{H}_{1}^{(0)}\right]_{i j k}=\frac{\partial^{2}\left[\varphi_{1}^{(0)}\right]_{i}}{\partial x_{j} \partial x_{k}}$, 
which acts as a linear operator mapping symmetric second-order tensors into vectors. Expanding the term $\sigma \circ \chi$ occurring in the surface action $\mathcal{S}_{\Sigma}$ to second order, we obtain

$\sigma \circ \chi=\epsilon\left\langle\nabla \sigma, \chi^{(1)}\right\rangle+\epsilon^{2}\left\{\left\langle\nabla \sigma, \chi^{(2)}\right\rangle+\frac{1}{2}\left\langle\nabla \nabla \sigma, \chi^{(1)} \otimes \chi^{(1)}\right\rangle\right\}+o\left(\epsilon^{3}\right)$,

and using the above results conclude that

$(\sigma \circ \chi)^{(1)}=\left\langle\mathbf{F}_{1}^{(0)-T} \cdot \nabla \sigma, \boldsymbol{\varphi}_{2}^{(1)}-\boldsymbol{\varphi}_{1}^{(1)}\right\rangle$,

$(\sigma \circ \chi)^{(2)}=-\left\langle\mathbf{F}_{1}^{(0)-T}\left[\nabla \sigma \otimes\left(\varphi_{2}^{(1)}-\boldsymbol{\varphi}_{1}^{(1)}\right)\right] \mathbf{F}^{(0)-T}, \mathbf{F}_{1}^{(1)}\right\rangle+\frac{1}{2}\left\langle\mathbf{S}_{1}^{(0)} \cdot\left(\boldsymbol{\varphi}_{2}^{(1)}-\boldsymbol{\varphi}_{1}^{(1)}\right), \boldsymbol{\varphi}_{2}^{(1)}-\boldsymbol{\varphi}_{1}^{(1)}\right\rangle$,

where we have defined for convenience

$\mathbf{S}_{1}^{(0)}=\mathbf{F}_{1}^{(0)-T}\left[\nabla \nabla \sigma-\mathbf{H}_{1}^{(0) T} \cdot\left(\mathbf{F}_{1}^{(0)-T} \cdot \nabla \sigma\right)\right] \mathbf{F}_{1}^{(0)-1}$,

with $\mathbf{H}_{1}^{(0) T}$ the transpose of the linear operator defined in eq. (104) which maps vectors into symmetric second-order tensors. In this manner, we have now arrived at the desired quadratic action for the fluid-solid body

$$
\begin{aligned}
\mathcal{S}^{(2)}= & \sum_{j=1}^{2} \int_{I} \int_{M_{j}}\left\{\frac{1}{2} \rho_{j}\left\|\mathbf{v}_{j}^{(1)}+\boldsymbol{\Omega} \times \boldsymbol{\varphi}_{j}^{(1)}\right\|^{2}-\frac{1}{2}\left\langle\boldsymbol{\Lambda}_{j} \cdot \mathbf{F}_{j}^{(1)}-\boldsymbol{S}_{j}, \mathbf{F}_{j}^{(1)}\right\rangle+\frac{1}{2} \rho_{j}\left\langle\boldsymbol{\gamma}_{j}^{(1)}, \boldsymbol{\varphi}_{j}^{(1)}\right\rangle\right\} \mathrm{d}^{3} \mathbf{x} \mathrm{d} t-\int_{I} \int_{\Sigma} \varpi^{(0)}\left\langle\mathbf{Q}_{1}^{(0)} \cdot\left(\boldsymbol{\varphi}_{2}^{(1)}-\boldsymbol{\varphi}_{1}^{(1)}\right), \mathbf{F}_{1}^{(1)}\right\rangle \mathrm{d} S \mathrm{~d} t \\
& +\frac{1}{2} \int_{I} \int_{\Sigma} \varpi^{(0)}\left\langle\mathbf{S}_{1}^{(0)} \cdot\left(\boldsymbol{\varphi}_{2}^{(1)}-\boldsymbol{\varphi}_{1}^{(1)}\right), \boldsymbol{\varphi}_{2}^{(1)}-\boldsymbol{\varphi}_{1}^{(1)}\right\rangle \mathrm{d} S \mathrm{~d} t+\int_{I} \int_{\Sigma} \varpi^{(1)}\left\langle\mathbf{F}_{1}^{(0)-T} \cdot \nabla \sigma, \boldsymbol{\varphi}_{2}^{(1)}-\boldsymbol{\varphi}_{1}^{(1)}\right\rangle \mathrm{d} S \mathrm{~d} t,
\end{aligned}
$$

where we have define a linear operator $\mathbf{Q}_{1}^{(0)}$ such that

$\mathbf{Q}_{1}^{(0)} \cdot\left(\boldsymbol{\varphi}_{2}^{(1)}-\boldsymbol{\varphi}_{1}^{(1)}\right)=\mathbf{F}_{1}^{(0)-T}\left[\nabla \sigma \otimes\left(\boldsymbol{\varphi}_{2}^{(1)}-\boldsymbol{\varphi}_{1}^{(1)}\right)\right] \mathbf{F}_{1}^{(0)-T}$

From this definition, it is clear that $\mathbf{Q}_{1}^{(0)}$ maps vectors into second-order tensors, and so is itself a third-order tensor. Within this action, $\varpi^{(1)}$ acts as a Lagrange multiplier field associated with the constraint

$\left\langle\mathbf{F}_{1}^{(0)-T} \cdot \nabla \sigma, \boldsymbol{\varphi}_{2}^{(1)}-\boldsymbol{\varphi}_{1}^{(1)}\right\rangle=0$

which states that the relative displacement $\varphi_{2}^{(1)}-\varphi_{1}^{(1)}$ must be tangential to the equilibrium fluid-solid boundary. We note that there are additional terms within the action associated with the fluid-solid boundary, and depending on the equilibrium value of the Lagrange multiplier field $\varpi^{(0)}$. As demonstrated later, the occurrence of these terms is consistent with Woodhouse \& Dahlen (1978), who incorporated similar surface integrals into their quadratic action in order to match the linearized continuity conditions they had obtained directly from Newton's third law of motion.

\subsubsection{Derivation of the linearized equations of motion in strong form}

To obtain the linearized equations of motion, we need to vary the action in eq. (108) with respect to each of the first-order fields $\left\{\boldsymbol{\varphi}_{1}^{(1)}, \boldsymbol{\varphi}_{2}^{(1)}, \varpi^{(1)}\right\}$. We have already noted that variation with respect to $\varpi^{(1)}$ leads to the linearized tangential slip constraint in eq. (110), and we can assume that this holds for the rest of the derivation. In taking the first variation of the remaining terms in the action, the only non-trivial step is associated the first integral over $\Sigma$. Using the chain rule and the definition of $\mathbf{Q}_{1}^{(0)}$ given above, we obtain

$$
\begin{aligned}
\varpi^{(0)}\left\langle\mathbf{F}_{1}^{(0)-T}\left[\nabla \sigma \otimes\left(\boldsymbol{\varphi}_{2}^{(1)}-\boldsymbol{\varphi}_{1}^{(1)}\right)\right] \mathbf{F}_{1}^{(0)-T}, \operatorname{Def} \delta \boldsymbol{\varphi}_{1}^{(1)}\right\rangle= & \operatorname{div}\left\{\varpi^{(0)}\left\langle\mathbf{F}_{1}^{(0)-T} \cdot \nabla \sigma, \delta \boldsymbol{\varphi}_{1}^{(1)}\right\rangle \mathbf{F}_{1}^{(0)-1} \cdot\left(\boldsymbol{\varphi}_{2}^{(1)}-\boldsymbol{\varphi}_{1}^{(1)}\right)\right\} \\
& -\left\langle\operatorname{Div}\left\{\varpi^{(0)} \mathbf{F}_{1}^{(0)-T}\left[\nabla \sigma \otimes\left(\boldsymbol{\varphi}_{2}^{(1)}-\boldsymbol{\varphi}_{1}^{(1)}\right)\right] \mathbf{F}_{1}^{(0)-T}\right\}, \delta \boldsymbol{\varphi}_{1}^{(1)}\right\rangle,
\end{aligned}
$$

and noting from eq. (110) that $\mathbf{F}_{1}^{(0)-1} \cdot\left[\boldsymbol{\varphi}_{2}^{(1)}-\boldsymbol{\varphi}_{1}^{(1)}\right]$ is a tangent vector field on $\Sigma$, we can use the divergence theorem on a closed surface (e.g. Abraham et al. 2012) to conclude that the integral over $\Sigma$ of the first term on the right-hand side of this identity is equal to zero. Given this result, it is then easy to see that the first variation of this quadratic action can be written

$$
\begin{aligned}
\delta \mathcal{S}^{(2)}= & -\sum_{j=1}^{2} \int_{I} \int_{M_{j}}\left\langle\rho_{j} \partial_{t} \mathbf{v}_{j}^{(1)}+2 \rho_{j} \boldsymbol{\Omega} \times \mathbf{v}_{j}^{(1)}+\rho_{j} \boldsymbol{\Omega} \times\left(\boldsymbol{\Omega} \times \boldsymbol{\varphi}_{j}^{(1)}\right)-\rho_{j} \boldsymbol{\gamma}_{j}^{(1)}-\operatorname{Div}\left(\mathbf{T}_{j}^{(1)}-\boldsymbol{S}_{j}\right), \delta \boldsymbol{\varphi}_{j}^{(1)}\right\rangle \mathrm{d}^{3} \mathbf{x} \mathrm{d} t-\int_{I} \int_{\Sigma}\left\langle\mathbf{t}_{1}^{(1)}, \delta \boldsymbol{\varphi}_{1}^{(1)}\right\rangle \mathrm{d} S \mathrm{~d} t \\
& +\int_{I} \int_{\Sigma}\left\langle\mathbf{t}_{2}^{(1)}, \delta \boldsymbol{\varphi}_{2}^{(1)}\right\rangle \mathrm{d} S \mathrm{~d} t-\int_{I} \int_{\partial M}\left\langle\mathbf{t}_{2}^{(1)}, \delta \boldsymbol{\varphi}_{2}^{(1)}\right\rangle \mathrm{d} S \mathrm{~d} t+\int_{I} \int_{\Sigma}\left\langle\operatorname{Div}\left\{\varpi^{(0)} \mathbf{F}_{1}^{(0)-T}\left[\nabla \sigma \otimes\left(\boldsymbol{\varphi}_{2}^{(1)}-\boldsymbol{\varphi}_{1}^{(1)}\right)\right] \mathbf{F}_{1}^{(0)-T}\right\}, \delta \boldsymbol{\varphi}_{1}^{(1)}\right\rangle \mathrm{d} S \mathrm{~d} t \\
& -\int_{I} \int_{\Sigma} \varpi^{(0)}\left\langle\left(\mathbf{F}_{1}^{(0)-T} \mathbf{F}_{1}^{(1) T} \mathbf{F}_{1}^{(0)-T}\right) \cdot \nabla \sigma, \delta \boldsymbol{\varphi}_{2}^{(1)}-\boldsymbol{\varphi}_{1}^{(1)}\right\rangle \mathrm{d} S \mathrm{~d} t+\int_{I} \int_{\Sigma} \varpi^{(0)}\left\langle\mathbf{S}_{1}^{(0)} \cdot\left(\boldsymbol{\varphi}_{2}^{(1)}-\boldsymbol{\varphi}_{1}^{(1)}\right), \delta \boldsymbol{\varphi}_{2}^{(1)}-\delta \boldsymbol{\varphi}_{1}^{(1)}\right\rangle \mathrm{d} S \mathrm{~d} t,
\end{aligned}
$$

where $\mathbf{T}_{j}^{(1)}=\boldsymbol{\Lambda}_{j} \cdot \mathbf{F}_{1}^{(1)}$ is the linearized first Piola-Kirchhoff stress tensor within $M_{j}$, and $\mathbf{t}_{j}^{(1)}=\left(\mathbf{T}_{j}^{(1)}-\mathbf{S}_{j}\right) \cdot \hat{\mathbf{n}}$ denotes the corresponding linearized referential traction including contributions from the stress glut. Setting the first variation equal to zero, we are led to the linearized 
momentum equation

$\rho_{j} \partial_{t} \mathbf{v}_{j}^{(1)}+2 \rho_{j} \boldsymbol{\Omega} \times \mathbf{v}_{j}^{(1)}+\rho_{j} \boldsymbol{\Omega} \times\left(\boldsymbol{\Omega} \times \boldsymbol{\varphi}_{j}^{(1)}\right)-\rho_{j} \boldsymbol{\gamma}_{j}^{(1)}-\operatorname{Div}\left(\mathbf{T}_{j}^{(1)}-\boldsymbol{S}_{j}\right)=\mathbf{0}$,

within each sub-body $M_{j}$, the linearized free-surface boundary condition $\mathbf{t}_{2}^{(1)}=\mathbf{0}$ on $\partial M$, and the following conditions on the referential fluid-solid boundary:

$$
\begin{aligned}
& \mathbf{t}_{1}^{(1)}-\operatorname{Div}\left\{\varpi^{(0)} \mathbf{F}_{1}^{(0)-T}\left[\nabla \sigma \otimes\left(\boldsymbol{\varphi}_{2}^{(1)}-\boldsymbol{\varphi}_{1}^{(1)}\right)\right] \mathbf{F}_{1}^{(0)-T}\right\}-\varpi^{(0)}\left(\mathbf{F}_{1}^{(0)-T} \mathbf{F}_{1}^{(1) T} \mathbf{F}_{1}^{(0)-T}\right) \cdot \nabla \sigma+\varpi^{(0)} \mathbf{S}^{(0)} \cdot\left(\boldsymbol{\varphi}_{2}^{(1)}-\boldsymbol{\varphi}_{1}^{(1)}\right)+\varpi^{(1)} \mathbf{F}_{1}^{(0)-T} \cdot \nabla \sigma=\mathbf{0} \\
& \mathbf{t}_{2}^{(1)}-\varpi^{(0)}\left(\mathbf{F}_{1}^{(0)-T} \mathbf{F}_{1}^{(1) T} \mathbf{F}_{1}^{(0)-T}\right) \cdot \nabla \sigma+\varpi^{(0)} \mathbf{S}^{(0)} \cdot\left(\boldsymbol{\varphi}_{2}^{(1)}-\boldsymbol{\varphi}_{1}^{(1)}\right)+\varpi^{(1)} \mathbf{F}_{1}^{(0)-T} \cdot \nabla \sigma=\mathbf{0}
\end{aligned}
$$

Combining these two identities, we obtain the linearized continuity conditions

$$
\begin{aligned}
\mathbf{t}_{2}^{(1)} & =\mathbf{t}_{1}^{(1)}-\operatorname{Div}\left\{\varpi^{(0)} \mathbf{F}_{1}^{(0)-T}\left[\nabla \sigma \otimes\left(\boldsymbol{\varphi}_{2}^{(1)}-\boldsymbol{\varphi}_{1}^{(1)}\right)\right] \mathbf{F}_{1}^{(0)-T}\right\} \\
& =\varpi^{(0)}\left(\mathbf{F}_{1}^{(0)-T} \mathbf{F}_{1}^{(1) T} \mathbf{F}_{1}^{(0)-T}\right) \cdot \nabla \sigma-\varpi^{(0)} \mathbf{S}^{(0)} \cdot\left(\boldsymbol{\varphi}_{2}^{(1)}-\boldsymbol{\varphi}_{1}^{(1)}\right)-\varpi^{(1)} \mathbf{F}_{1}^{(0)-T} \cdot \nabla \sigma,
\end{aligned}
$$

which can also be obtained through careful linearization of eq. (77). Within these equations, we note that the equilibrium stress field and the equilibrium configuration occur explicitly within the continuity conditions at the fluid-solid boundary. Again, this is consistent with the results of Woodhouse \& Dahlen (1978). These parameters do, of course, also influence the momentum equations, though here the dependence is contained implicitly within the elastic tensor, the referential gravitational acceleration and the centrifugal potential.

\subsubsection{Derivation of the linearized equations of motion in weak form}

An alternative variational principle for the linearized equations of motion can be obtained by restricting the configuration space of the problem to displacement fields $\left\{\boldsymbol{\varphi}_{1}^{(1)}, \boldsymbol{\varphi}_{2}^{(1)}\right\}$ satisfying an appropriate linearized form of the tangential slip constraint. In fact, we stated such a constraint in eq. (102), but it will be useful to reformulate this condition as

$\varphi_{2}^{(1)}-\varphi_{1}^{(1)}=\mathbf{F}_{1}^{(0)} \cdot \chi^{(1)}$,

for $(\mathbf{x}, t) \in \Sigma \times I$, where it is understood that $\chi^{(1)}$ is an arbitrary tangential vector field on $\Sigma$. Having imposed this constraint on the configuration space, we can write the quadratic action for the linearized problem as

$$
\begin{aligned}
\mathcal{S}^{(2)}= & \sum_{j=1}^{2} \int_{I} \int_{M_{j}}\left\{\frac{1}{2} \rho_{j}\left\|\mathbf{v}_{j}^{(1)}+\mathbf{\Omega} \times \boldsymbol{\varphi}_{j}^{(1)}\right\|^{2}-\frac{1}{2}\left\langle\boldsymbol{\Lambda}_{j} \cdot \mathbf{F}_{j}^{(1)}-\boldsymbol{S}_{j}, \mathbf{F}_{j}^{(1)}\right\rangle+\frac{1}{2} \rho_{j}\left\langle\boldsymbol{\gamma}_{j}^{(1)}, \boldsymbol{\varphi}_{j}^{(1)}\right\rangle\right\} \mathrm{d}^{3} \mathbf{x} \mathrm{d} t \\
& -\int_{I} \int_{\Sigma} \varpi^{(0)}\left\langle\mathbf{Q}_{1}^{(0)} \cdot\left(\boldsymbol{\varphi}_{2}^{(1)}-\boldsymbol{\varphi}_{1}^{(1)}\right), \mathbf{F}_{1}^{(1)}\right\rangle \mathrm{d} S \mathrm{~d} t+\frac{1}{2} \int_{I} \int_{\Sigma} \varpi^{(0)}\left\langle\mathbf{S}_{1}^{(0)} \cdot\left(\boldsymbol{\varphi}_{2}^{(1)}-\boldsymbol{\varphi}_{1}^{(1)}\right), \boldsymbol{\varphi}_{2}^{(1)}-\boldsymbol{\varphi}_{1}^{(1)}\right\rangle \mathrm{d} S \mathrm{~d} t,
\end{aligned}
$$

which is identical to eq. (108) except for the absence of the now unnecessary term associated with the Lagrange multiplier $\varpi^{(1)}$. By varying this action with respect to $\varphi_{1}^{(1)}$ and $\varphi_{2}^{(1)}$ subject to the above linearized tangential slip constraint, we would again obtain the linearized equations of motion for the body. We will, however, not carry out this derivation in full, but instead express the stationarity condition in following intermediate form:

$$
\begin{aligned}
& -\sum_{j=1}^{2} \int_{I} \int_{M_{j}}\left\{\rho_{j}\left\langle\partial_{t} \mathbf{v}_{j}^{(1)}+2 \boldsymbol{\Omega} \times \mathbf{v}_{j}^{(1)}+\boldsymbol{\Omega} \times\left(\boldsymbol{\Omega} \times \boldsymbol{\varphi}_{j}^{(1)}\right)-\boldsymbol{\gamma}_{j}^{(1)}, \delta \boldsymbol{\varphi}_{j}^{(1)}\right\rangle+\left\langle\mathbf{T}_{j}^{(1)}-\boldsymbol{S}_{j}, \operatorname{Def} \delta \boldsymbol{\varphi}_{j}^{(1)}\right\rangle\right\} \mathrm{d}^{3} \mathbf{x} \mathrm{d} t \\
& -\int_{I} \int_{\Sigma} \varpi^{(0)}\left\langle\mathbf{Q}_{1}^{(0)} \cdot\left(\boldsymbol{\varphi}_{2}^{(1)}-\boldsymbol{\varphi}_{1}^{(1)}\right), \operatorname{Def} \delta \boldsymbol{\varphi}_{1}^{(1)}\right\rangle \mathrm{d} S \mathrm{~d} t-\int_{I} \int_{\Sigma} \varpi^{(0)}\left\langle\operatorname{Def} \boldsymbol{\varphi}_{1}^{(1)}, \mathbf{Q}_{1}^{(0)} \cdot\left(\delta \boldsymbol{\varphi}_{2}^{(1)}-\delta \boldsymbol{\varphi}_{1}^{(1)}\right)\right\rangle \mathrm{d} S \mathrm{~d} t \\
& +\int_{I} \int_{\Sigma} \varpi^{(0)}\left\langle\mathbf{S}_{1}^{(0)} \cdot\left(\boldsymbol{\varphi}_{2}^{(1)}-\boldsymbol{\varphi}_{1}^{(1)}\right), \delta \boldsymbol{\varphi}_{2}^{(1)}-\delta \boldsymbol{\varphi}_{1}^{(1)}\right\rangle \mathrm{d} S \mathrm{~d} t=0,
\end{aligned}
$$

where we have only carried out the temporal integrations by parts. This result must hold for all $\left\{\delta \varphi_{1}, \delta \varphi_{2}\right\}$ subject to the constraint in eq. (117), and as there are no time-derivatives of the variations in the above expression, it is equivalent to the condition

$$
\begin{aligned}
& -\sum_{j=1}^{2} \int_{M_{j}}\left\{\rho_{j}\left\langle\partial_{t} \mathbf{v}_{j}^{(1)}+2 \boldsymbol{\Omega} \times \mathbf{v}_{j}^{(1)}+\boldsymbol{\Omega} \times\left(\boldsymbol{\Omega} \times \boldsymbol{\varphi}_{j}^{(1)}\right)-\boldsymbol{\gamma}_{j}^{(1)}, \delta \boldsymbol{\varphi}_{j}^{(1)}\right\rangle+\left\langle\mathbf{T}_{j}^{(1)}-\boldsymbol{S}_{j}, \operatorname{Def} \delta \boldsymbol{\varphi}_{j}^{(1)}\right\rangle\right\} \mathrm{d}^{3} \mathbf{x} \\
& -\int_{\Sigma} \varpi^{(0)}\left\langle\mathbf{Q}_{1}^{(0)} \cdot\left(\boldsymbol{\varphi}_{2}^{(1)}-\boldsymbol{\varphi}_{1}^{(1)}\right), \operatorname{Def} \delta \boldsymbol{\varphi}_{1}^{(1)}\right\rangle \mathrm{d} S-\int_{\Sigma} \varpi^{(0)}\left\langle\operatorname{Def} \boldsymbol{\varphi}_{1}^{(1)}, \mathbf{Q}_{1}^{(0)} \cdot\left(\delta \boldsymbol{\varphi}_{2}^{(1)}-\delta \boldsymbol{\varphi}_{1}^{(1)}\right)\right\rangle \mathrm{d} S \\
& +\int_{\Sigma} \varpi^{(0)}\left\langle\mathbf{S}_{1}^{(0)} \cdot\left(\boldsymbol{\varphi}_{2}^{(1)}-\boldsymbol{\varphi}_{1}^{(1)}\right), \delta \boldsymbol{\varphi}_{2}^{(1)}-\delta \boldsymbol{\varphi}_{1}^{(1)}\right\rangle \mathrm{d} S=0,
\end{aligned}
$$


for all time-independent variations $\left\{\delta \varphi_{1}, \delta \varphi_{2}\right\}$. Eq. (120) is a statement of the weak form of the linearized equations of motions. It is this formulation of the problem which is required within numerical methods based on Galerkin expansions, including both normal mode coupling and finite-element calculations.

\subsubsection{Reduction to the results of Woodhouse \& Dahlen (1978)}

The linearized equations of motion obtained in Section 3.3.2 are valid with respect to an arbitrary reference configuration. It is, however, usual to work with the reference configuration such that particles are labelled by their position at equilibrium, and hence that $\varphi_{j}^{(0)}(\mathbf{x})=\mathbf{x}$. Following AC16, such a reference configuration will be called 'natural', and it follows easily that in this case the quadratic action in eq. (108) reduces to

$$
\begin{aligned}
\mathcal{S}^{(2)}= & \sum_{j=1}^{2} \int_{I} \int_{M_{j}}\left\{\frac{1}{2} \rho_{j}\left\|\mathbf{v}_{j}^{(1)}+\boldsymbol{\Omega} \times \boldsymbol{\varphi}_{j}^{(1)}\right\|^{2}-\frac{1}{2}\left\langle\boldsymbol{\Lambda}_{j} \cdot \mathbf{F}_{j}^{(1)}, \mathbf{F}_{j}^{(1)}\right\rangle+\frac{1}{2} \rho_{j}\left\langle\boldsymbol{\gamma}_{j}^{(1)}, \boldsymbol{\varphi}_{j}^{(1)}\right\rangle\right\} \mathrm{d}^{3} \mathbf{x} \mathrm{d} t \\
& -\int_{I} \int_{\Sigma} \varpi^{(0)}\left\langle\nabla \sigma \otimes\left(\boldsymbol{\varphi}_{2}^{(1)}-\boldsymbol{\varphi}_{1}^{(1)}\right), \mathbf{F}_{1}^{(1)}\right\rangle \mathrm{d} S \mathrm{~d} t+\frac{1}{2} \int_{I} \int_{\Sigma} \varpi^{(0)}\left\langle\nabla \nabla \sigma \cdot\left(\boldsymbol{\varphi}_{2}^{(1)}-\boldsymbol{\varphi}_{1}^{(1)}\right), \boldsymbol{\varphi}_{2}^{(1)}-\boldsymbol{\varphi}_{1}^{(1)}\right\rangle \mathrm{d} S \mathrm{~d} t \\
& +\int_{I} \int_{\Sigma} \varpi^{(1)}\left\langle\nabla \sigma, \boldsymbol{\varphi}_{2}^{(1)}-\boldsymbol{\varphi}_{1}^{(1)}\right\rangle \mathrm{d} S \mathrm{~d} t
\end{aligned}
$$

where we note that $\boldsymbol{\gamma}_{j}^{(1)}$ in eq. (99) now takes the simpler form

$\boldsymbol{\gamma}_{j}^{(1)}(\mathbf{x})=-\sum_{k=1}^{2} \int_{M_{k}} \rho_{k}\left(\mathbf{x}^{\prime}\right)(\nabla \nabla \Gamma)\left(\mathbf{x}-\mathbf{x}^{\prime}\right) \cdot\left[\boldsymbol{\varphi}_{j}^{(1)}(\mathbf{x})-\boldsymbol{\varphi}_{k}^{(1)}\left(\mathbf{x}^{\prime}\right)\right] \mathrm{d}^{3} \mathbf{x}^{\prime}$,

and in this section we neglect the presence of a stress glut. The corresponding equations of motion are readily obtained from the general case discussed above, and we will show that these equations are equivalent to the results of Woodhouse \& Dahlen (1978). In fact, the only non-trivial step in doing this relates to the continuity conditions at fluid-solid boundaries. Using eq. (116), we see that the continuity conditions we obtained reduce to the form

$$
\begin{aligned}
\mathbf{t}_{2}^{(1)}= & \mathbf{t}_{1}^{(1)}-\operatorname{Div}\left[\varpi^{(0)} \nabla \sigma \otimes\left(\boldsymbol{\varphi}_{2}^{(1)}-\boldsymbol{\varphi}_{1}^{(1)}\right)\right] \\
& =\varpi^{(0)} \mathbf{F}_{1}^{(1) T} \cdot \nabla \sigma-\varpi^{(0)} \nabla \nabla \sigma \cdot\left(\boldsymbol{\varphi}_{2}^{(1)}-\boldsymbol{\varphi}_{1}^{(1)}\right)-\varpi^{(1)} \nabla \sigma .
\end{aligned}
$$

Beginning with the first of these equalities, we use the product rule to obtain

$\mathbf{t}_{2}=\mathbf{t}_{1}-\operatorname{div}^{\Sigma}\left[\varpi^{(0)}\left(\varphi_{2}^{(1)}-\varphi_{1}^{(1)}\right)\right] \nabla \sigma-\varpi^{(0)} \nabla \nabla \sigma \cdot\left(\varphi_{2}^{(1)}-\varphi_{1}^{(1)}\right)$

where the tangential divergence operator $\operatorname{div}^{\Sigma}$ (e.g. Dahlen \& Tromp 1998, appendix A) has been introduced as $\varpi^{(0)}\left(\varphi_{2}^{(1)}-\varphi_{1}^{(1)}\right)$ is a tangential vector field on $\Sigma$. We next observe that

$\nabla^{\Sigma}\left\langle\nabla \sigma, \boldsymbol{\varphi}_{2}^{(1)}-\boldsymbol{\varphi}_{1}^{(1)}\right\rangle=\left(\nabla^{\Sigma} \nabla \sigma\right) \cdot\left(\boldsymbol{\varphi}_{2}^{(1)}-\boldsymbol{\varphi}_{1}^{(1)}\right)+\left[\nabla^{\Sigma}\left(\boldsymbol{\varphi}_{2}^{(1)}-\boldsymbol{\varphi}_{1}^{(1)}\right)\right] \cdot \nabla \sigma=\mathbf{0}$,

because $\varphi_{2}^{(1)}-\varphi_{1}^{(1)}$ is tangent to $\Sigma$. Here, $\nabla^{\Sigma}$ denotes the tangential gradient operator on $\Sigma$ whose action is defined on a scalar fields $f$ by

$\nabla^{\Sigma} f=\nabla f-\langle\nabla f, \hat{\mathbf{n}}\rangle \hat{\mathbf{n}}$

and can be extended to a vector field $\mathbf{w}$ through

$\left(\nabla^{\Sigma} \mathbf{w}\right)_{i j}=\frac{\partial w_{j}}{\partial x_{i}}-n_{i} n_{k} \frac{\partial w_{j}}{\partial x_{k}}$.

We can always choose $\sigma$ such that $\left.\nabla \sigma\right|_{\Sigma}=\hat{\mathbf{n}}$, and $\|\nabla \sigma\|=1$ in some open neighbourhood of $\Sigma$. For example, when $\Sigma$ is a sphere of radius $a$, we take $\sigma(\mathbf{x})=\|\mathbf{x}\|-a$. It follows that $\nabla \nabla \sigma=\nabla^{\Sigma} \nabla \sigma$, and from eq. (125) we arrive at

$\nabla \nabla \sigma \cdot\left(\varphi_{2}^{(1)}-\varphi_{1}^{(1)}\right)=-\left[\nabla^{\Sigma}\left(\varphi_{2}^{(1)}-\varphi_{1}^{(1)}\right)\right] \cdot \hat{\mathbf{n}}$.

Putting these results together, we conclude that the following vector

$\boldsymbol{\tau}^{(1)}=\mathbf{t}^{(1)}+\operatorname{div}^{\Sigma}\left(\varpi^{(0)} \boldsymbol{\varphi}^{(1)}\right) \hat{\mathbf{n}}-\varpi^{(0)}\left(\nabla^{\Sigma} \boldsymbol{\varphi}^{(1)}\right) \cdot \hat{\mathbf{n}}$

is continuous across fluid-solid boundaries. Noting that $-\varpi^{(0)}$ is equal to Woodhouse and Dahlen's variable $\pi_{0}$, we see the continuity of $\boldsymbol{\tau}^{(1)}$ is equivalent to the first equality within eq. (24) in their paper. Woodhouse \& Dahlen (1978) further showed that the vector $\boldsymbol{\tau}^{(1)}$ should always be parallel to the normal $\hat{\mathbf{n}}$. To see that this holds, we use eq. (128) to write the second equality in eq. (123) in the form

$\mathbf{t}_{2}^{(1)}-\varpi^{(0)}\left(\nabla^{\Sigma} \boldsymbol{\varphi}_{2}^{(1)}\right) \cdot \hat{\mathbf{n}}=\varpi^{(0)}\left[\nabla \boldsymbol{\varphi}_{1}^{(1)}-\nabla^{\Sigma} \boldsymbol{\varphi}_{1}^{(1)}\right] \cdot \hat{\mathbf{n}}-\varpi^{(1)} \hat{\mathbf{n}}$, 
and using the defining property of the tangential gradient operator we obtain

$\left[\nabla \boldsymbol{\varphi}_{1}^{(1)}-\nabla^{\Sigma} \boldsymbol{\varphi}_{1}^{(1)}\right] \cdot \hat{\mathbf{n}}=\left\langle\operatorname{Def} \boldsymbol{\varphi}_{1}^{(1)} \cdot \hat{\mathbf{n}}, \hat{\mathbf{n}}\right\rangle \hat{\mathbf{n}}$.

From these results and the definition in eq. (129), we have shown that

$\boldsymbol{\tau}_{2}^{(1)}=\left\{\operatorname{div}^{\Sigma}\left(\varpi^{(0)} \varphi^{(1)}\right)+\varpi^{(0)}\left\langle\operatorname{Def} \varphi_{1}^{(1)} \cdot \hat{\mathbf{n}}, \hat{\mathbf{n}}\right\rangle-\varpi^{(1)}\right\} \hat{\mathbf{n}}$,

and so $\boldsymbol{\tau}^{(1)}$ is indeed always normal to the boundary.

\subsubsection{Linearized equations of motion in the absence of gravitational forces}

For seismic application at high frequencies, it can be shown that terms in the equations of motion associated with gravitation become negligible (e.g. Dahlen \& Tromp 1998, section 4.3.5). In the absence of gravitation forces, it is usually assumed that the equilibrium configuration of the body is stress-free, and so from eq. (92) we obtain $\varpi^{(0)}=0$. The quadratic Lagrangian for the problem given in eq. (108) then reduces to the simpler form

$\mathcal{S}^{(2)}=\sum_{j=1}^{2} \int_{I} \int_{M_{j}}\left\{\frac{1}{2} \rho_{j}\left\|\mathbf{v}_{j}^{(1)}+\boldsymbol{\Omega} \times \boldsymbol{\varphi}_{j}^{(1)}\right\|^{2}-\frac{1}{2}\left\langle\boldsymbol{\Lambda}_{j} \cdot \mathbf{F}_{j}^{(1)}-\mathfrak{S}_{j}, \mathbf{F}_{j}^{(1)}\right\rangle\right\} \mathrm{d}^{3} \mathbf{x} \mathrm{d} t$,

where we have chosen to imposed the linearized tangential slip constraint in eq. (117) explicitly within the configuration space for the problem. Here we see that there is now no surface integral terms occurring in the action associated with tangential slip, this again being consistent with the results of Woodhouse \& Dahlen (1978).

\subsection{Particle relabelling transformations}

Here, we describe briefly how particle relabelling transformations discussed above carry over to the linearized problem. These transformations take exactly the same form as discussed in section 4.2 of AC16, and we will just summarize the key results. First, the equilibrium field $\varpi^{(0)}$ occurring in eq. (108) transforms in the following manner:

$\tilde{\varpi}^{(0)}(\mathbf{x})=J_{\left.\xi\right|_{\tilde{\Sigma}}}(\mathbf{x}) \varpi^{(0)}[\boldsymbol{\xi}(\mathbf{x})]$,

which follows immediately from eq. (134). The referential density $\rho$ is transformed as

$\tilde{\rho}(\mathbf{x})=J_{\xi}(\mathbf{x}) \rho[\xi(\mathbf{x})]$,

which is identical to the transformation for finite deformations. Using the transformation property of the strain energy function given within eq. (83), it can be shown through a simple calculation that the components of the elastic tensor transform as

$\tilde{\Lambda}_{i j k l}(\mathbf{x})=J_{\xi}(\mathbf{x})\left[\mathbf{F}_{\xi}(\mathbf{x})^{-1}\right]_{j m}\left[\mathbf{F}_{\xi}(\mathbf{x})^{-1}\right]_{l n} \Lambda_{i m k n}[\xi(\mathbf{x})]$.

In this manner, we can use particle relabelling transformations to relate the material parameters of the body between any two different choices of reference configuration.

\section{APPLiCATIONS TO MODE COUPLING CALCULATIONS}

\subsection{A streamlined notation}

In discussing the practical applications of our results to normal mode coupling calculations, it will be helpful to introduce a simpler, though less systematic, notation for the various fields involved. Mode coupling calculations are based on the weak formulation of the linearized equations of motion as given by eq. (120), and we note that the linearized tangential slip constraint in eq. (117) must be explicitly enforced within this description of the problem. Describing these new notations will entail a little repetition, but this is done for the convenience of those rejoining the paper at this point.

For the equilibrium mapping $\varphi^{(0)}$ that carries points $\mathbf{x} \in M$ in the reference body to their position at equilibrium, we will now write $\boldsymbol{\Phi}$. The time-dependent linearized motion of the body about equilibrium will be written $\mathbf{u}$ instead of the earlier $\varphi^{(1)}$. The associated first-order perturbation to the referential gravitational acceleration $\boldsymbol{\gamma}^{(1)}$ is now written $\boldsymbol{\gamma}$. Variations of the linearized motion occurring within the weak formulation of the problem in eq. (120) are now denoted by $\mathbf{w}$ and not the earlier $\delta \varphi^{(1)}$. The equilibrium value $\varpi^{(0)}$ of the Lagrange multiplier field associated with the tangential slip constraint is now written $\varpi$. Finally tensors $\mathbf{S}_{1}^{(0)}$ and $\mathbf{Q}_{1}^{(0)}$ defined in eqs (107) and (109), respectively, and associated with the fluid-solid boundary conditions will be written simply as $\mathbf{S}$ and $\mathbf{Q}$. 
Given these notational changes, we now can rewrite the weak form of the problem from eq. (120) in the simplified manner

$$
\begin{aligned}
& -\int_{M}\left\{\rho\left\langle\partial_{t}^{2} \mathbf{u}+2 \boldsymbol{\Omega} \times \partial_{t} \mathbf{u}+\boldsymbol{\Omega} \times(\boldsymbol{\Omega} \times \mathbf{u})-\boldsymbol{\gamma}, \mathbf{w}\right\rangle+\langle\boldsymbol{\Lambda} \cdot \operatorname{Def} \mathbf{u}-\boldsymbol{S}, \operatorname{Def} \mathbf{w}\rangle\right\} \mathrm{d}^{3} \mathbf{x} \\
& -\int_{\Sigma} \varpi\left\langle\mathbf{Q} \cdot\left(\mathbf{u}_{2}-\mathbf{u}_{1}\right), \operatorname{Def} \mathbf{w}_{1}\right\rangle \mathrm{d} S-\int_{\Sigma} \varpi\left\langle\operatorname{Def} \mathbf{u}_{1}, \mathbf{Q} \cdot\left(\mathbf{w}_{2}-\mathbf{w}_{1}\right)\right\rangle \mathrm{d} S+\int_{\Sigma} \varpi\left\langle\mathbf{S} \cdot\left(\mathbf{u}_{2}-\mathbf{u}_{1}\right), \mathbf{w}_{2}-\mathbf{w}_{1}\right\rangle \mathrm{d} S=0,
\end{aligned}
$$

which is to hold for variations of the test functions $\mathbf{w}$, and it is understood that both the displacement field $\mathbf{u}$ and the test function $\mathbf{w}$ are subject to the linearized tangential slip constraint of the form

$\left\langle\left(\operatorname{Def} \boldsymbol{\Phi}_{1}\right)^{-1} \cdot\left(\mathbf{u}_{2}-\mathbf{u}_{1}\right), \hat{\mathbf{n}}\right\rangle=0$,

on the referential fluid-solid boundary $\Sigma$. Note that in writing this simplified version of the weak form, we have retained the use of subscripts to denote the subregion of the body a field is defined in, but only included these where needed within the surface integral terms. The terms $\varpi, \mathbf{S}$ and $\mathbf{Q}$ within the problem are determined by the body's equilibrium configuration, and act as parameters within the linearized equations of motion.

\subsection{Derivation of the mode coupling equations}

Suppose we start with a geometrically aspherical earth model in which we wish to calculate synthetic-free oscillation spectra. First, we perform a suitable particle relabelling transformation to obtain a reference configuration for the body which is geometrically spherical. The practical details of such a transformation will be discussed elsewhere. Having done this, the material parameters $(\rho, \boldsymbol{\Lambda})$ of the body are now defined relative to this geometrically spherical reference body, while any boundary topography is contained implicitly within the volumetric parameter $\boldsymbol{\Phi}$. In practice, the laterally heterogeneous model under consideration will represent a mild perturbation away from some spherically symmetric model whose eigenfunctions and eigenfrequencies can be determined. We shall write $\mathbf{u}_{n}$ for these reference eigenfunctions, and note that across the fluid-solid boundary they satisfy the tangential slip condition

$$
\left\langle\left[\mathbf{u}_{n}\right]_{-}^{+}, \hat{\mathbf{n}}\right\rangle=0
$$

where $[\cdot]_{-}^{+}$denotes the jump in a quantity in the direction of the outward unit normal. Such eigenfunctions will be assumed to form a complete basis for vector fields in the reference body satisfying this continuity condition.

The tangential slip constraint in eq. (138) required in the displacement field and test functions in our problem is not, however, the same as that satisfied by the reference eigenfunctions. To proceed, we introduce a smooth function B : $M \rightarrow$ GL(3) taking values in the general linear group such that

$\left.\mathbf{B}\right|_{\Sigma}=\operatorname{Def} \boldsymbol{\Phi}_{1}$

In particular, the support of $\mathbf{B}-\mathbf{1}$ can always be chosen arbitrarily close to $\Sigma$. Defining a new set of functions by $\hat{\mathbf{u}}_{n}=\mathbf{B} \cdot \mathbf{u}_{n}$, we obtain a complete basis for vector fields in $M$ satisfying the correct tangential slip condition at the fluid-solid boundary. We can now expand the displacement field in the laterally heterogeneous model as

$\mathbf{u}(\mathbf{x}, t)=\sum_{n} u_{n}(t) \hat{\mathbf{u}}_{n}(\mathbf{x})$,

where the $u_{n}(t)$ are expansion coefficients to be determined. This expansion can be substituted into the weak form of the problem in eq. (137), and by taking as test function $\mathbf{w}=\hat{\mathbf{u}}_{n^{\prime}}$ for arbitrary values of $n^{\prime}$ we arrive the following system of mode coupling equations

$\mathbf{M u ̈}+\mathbf{W} \dot{\mathbf{u}}+\mathbf{H u}=\mathbf{f}$

where $\mathbf{u}$ is an infinite-dimensional vector of expansion coefficients, while $\mathbf{M}, \mathbf{W}$ and $\mathbf{H}$ are infinite-dimensional matrices whose elements take the form

$\mathbf{M}_{n^{\prime} n}=\int_{M} \rho\left\langle\hat{\mathbf{u}}_{n}, \hat{\mathbf{u}}_{n^{\prime}}\right\rangle \mathrm{d}^{3} \mathbf{x}$

$\mathrm{W}_{n^{\prime} n}=\int_{M} 2 \rho\left\langle\boldsymbol{\Omega} \times \hat{\mathbf{u}}_{n}, \hat{\mathbf{u}}_{n^{\prime}}\right\rangle \mathrm{d}^{3} \mathbf{x}$

$$
\begin{aligned}
\mathrm{H}_{n^{\prime} n}= & \int_{M}\left\{\rho\left\langle\boldsymbol{\Omega} \times\left(\boldsymbol{\Omega} \times \hat{\mathbf{u}}_{n}\right)-\boldsymbol{\gamma}_{n}, \hat{\mathbf{u}}_{n^{\prime}}\right\rangle+\left\langle\boldsymbol{\Lambda} \cdot \operatorname{Def} \hat{\mathbf{u}}_{n}, \operatorname{Def} \hat{\mathbf{u}}_{n^{\prime}}\right\rangle\right\} \mathrm{d}^{3} \mathbf{x}+\int_{\Sigma} \varpi\left\langle\mathbf{Q} \cdot\left[\hat{\mathbf{u}}_{n}\right]_{-}^{+},\left.\operatorname{Def} \hat{\mathbf{u}}_{n^{\prime}}\right|_{-}\right\rangle \mathrm{d} S \\
& +\int_{\Sigma} \varpi\left\langle\left.\operatorname{Def} \hat{\mathbf{u}}_{n}\right|_{-}, \mathbf{Q} \cdot\left[\hat{\mathbf{u}}_{n^{\prime}}\right]_{-}^{+}\right\rangle \mathrm{d} S-\int_{\Sigma} \varpi\left\langle\mathbf{S} \cdot\left[\hat{\mathbf{u}}_{n}\right]_{-}^{+},\left[\hat{\mathbf{u}}_{n^{\prime}}\right]_{-}^{+}\right\rangle \mathrm{d} S,
\end{aligned}
$$


where $\boldsymbol{\gamma}_{n}$ denotes the referential gravitational acceleration perturbation associated with the basis vector $\hat{\mathbf{u}}_{n}$, and we note that Def $\hat{\mathbf{u}}_{n^{\prime}} \mid-$ means the quantity Def $\hat{\mathbf{u}}_{n^{\prime}}$ evaluated on the lower side of $\Sigma$. Finally, the force term in the mode coupling equations is given by

$\mathbf{f}_{n^{\prime}}=\int_{M}\left\langle\mathfrak{S}\right.$, Def $\left.\hat{\mathbf{u}}_{n^{\prime}}\right\rangle \mathrm{d}^{3} \mathbf{x}$

The mass matrix $\mathbf{M}$ is obviously symmetric and positive-definite, while the Coriolis matrix $\mathbf{W}$ is antisymmetric. Finally, the stiffness matrix $\mathbf{H}$ is almost trivially symmetric, with a simple calculation involving the term $\boldsymbol{\gamma}_{n}$ using eq. (99) showing that this is indeed the case. The form of the coupling equations in eq. (142) is identical to those considered in, for example, Al-Attar et al. (2012), and so the same numerical methods can be used in their solution. The practical differences to previous work on mode coupling are that (i) these new matrix elements account for aspherical boundary topography exactly and (ii) the effects of self-gravitation are fully incorporated, this being in contrast to previous studies that retained terms only to first order in aspherical density perturbations. Following such a mapping into a spherical reference body, it is notable, however, that the elastic tensor will loose some of its usual symmetries (see section 4.3 of AC16 for a full discussion).

\section{CONCLUSIONS}

In this paper, we have shown how Hamilton's principle can be formulated within a self-gravitating fluid-solid simple hyperelastic body relative to an arbitrary reference configuration. To our knowledge, this is an original result, and is of some independent interest. Using this variational principle, we have obtained the exact and linearized equations of motion, including the weak formulation of the latter required within normal mode coupling calculations and other numerical approaches based on Galerkin methods. Because we have formulated the problem relative to an arbitrary reference body, we can map any topography on internal and external boundaries into effective volumetric heterogeneity in an exact manner within a geometrically spherical reference body. Having done this, expansion of the displacement vector in the eigenfunctions of a spherically symmetric reference body is permissible (subject to the minor modifications discussed above), and in this manner it will be possible to exactly incorporate boundary topography into normal mode coupling calculations for the first time. The numerical implementation of this approach will be described in a forthcoming work. Beyond this specific application, the methods of this paper have potential application to a range of other problems that currently rely on perturbation theory to account for the asphericity of the Earth or of Earth-like planets. Here, we mention in particular the recent work of Lock \& Stewart (2017) which suggests that early stages of planetary evolution may be characterized by rapidly rotating and highly flattened shapes. The dynamics of such strongly aspherical bodies cannot be sensibly modelled using boundary perturbation theory, but the methods of this paper should be applicable.

\section{ACKNOWLEDGEMENTS}

We thank John Woodhouse for his influence on this work. We would also like to thank the editor Duncan Agnew along with reviewers Guust Nolet and Andreas Fichtner for their careful reading and helpful comments. The research leading to these results has received partial funding from the European Research Council (ERC) under the European Union's Seventh Framework Programme (FP/2007-2013) grant agreement number 320639 (iGEO). OC is supported by an NERC studentship with a CASE award from the British Antarctic Survey. APV acknowledges support from the Australian Research Council (grant DE180100040), Geoscience Australia, and The Research School of Earth Sciences at ANU.

\section{REFERENCES}

Abraham, R., Marsden, J.E. \& Marsden, J.E., 1978. Foundations of Mechanics, Vol. 36, Benjamin/Cummings Publishing Company Reading.

Abraham, R., Marsden, J.E. \& Ratiu, T., 2012. Manifolds, Tensor Analysis, and Applications, Vol. 75, Springer Science \& Business Media.

Agmon, S., 1962. On the eigenfunctions and on the eigenvalues of general elliptic boundary value problems, Commun. Pure appl. Math., 15(2), 119-147.

Agmon, S., Douglis, A. \& Nirenberg, L., 1959. Estimates near the boundary for solutions of elliptic partial differential equations satisfying general boundary conditions. i, Commun. Pure appl. Math., 12(4), 623-727.

Agmon, S., Douglis, A. \& Nirenberg, L., 1964. Estimates near the boundary for solutions of elliptic partial differential equations satisfying general boundary conditions ii, Commun. Pure appl. Math., 17(1), 35-92.

Akbarashrafi, F., Al-Attar, D., Deuss, A., Trampert, J. \& Valentine, A.P., 2018. Exact free oscillation spectra, splitting functions and the resolvability of earth's density structure, Geophys. J. Int., 213(1), 58-76.

Al-Attar, D., 2011. Theoretical problems in global seismology and geodynamics, D.Phil. thesis, University of Oxford.

Al-Attar, D. \& Crawford, O., 2016. Particle relabelling transformations in elastodynamics, Geophys. J. Int., 205(1), 575-593.
Al-Attar, D. \& Woodhouse, J.H., 2010. On the parametrization of equilibrium stress fields in the earth, Geophys. J. Int., 181(1), 567-576.

Al-Attar, D., Woodhouse, J.H. \& Deuss, A., 2012. Calculation of normal mode spectra in laterally heterogeneous earth models using an iterative direct solution method, Geophys. J. Int., 189(2), 1038-1046.

Antman, S.S., 2005. Problems in nonlinear elasticity, in Nonlinear Problems of Elasticity, pp. 513-584, Springer.

Backus, G. \& Mulcahy, M., 1976. Moment tensors and other phenomenological descriptions of seismic sources - I. Continuous displacements, Geophys. J. Int., 46(2), 341-361.

Backus, G.E., 1967. Converting vector and tensor equations to scalar equations in spherical coordinates, Geophys. J. Int., 13(1-3), 71-101.

Boore, D.M., 1972. Finite difference methods for seismic wave propagation in heterogeneous materials, Methods Comput. Phys., 11, 1-37.

Brazda, K., de Hoop, M.V. \& Hoermann, G., 2017. Variational formulation of the earth's elastic-gravitational deformations under low regularity conditions, preprint arXiv:1702.04741.

Browder, F.E., 1961. On the spectral theory of elliptic differential operators. i, Math. Ann., 142(1), 22-130.

Chaljub, E. \& Valette, B., 2004. Spectral element modelling of threedimensional wave propagation in a self-gravitating earth with an arbitrarily stratified outer core, Geophys. J. Int., 158(1), 131-141. 
Cisternas, A. \& Jobert, G., 1977. Extension of matrix-methods to structures with slightly irregular stratification, J. Geophys., 43(1-2), 59-74.

Dahlen, F., 1969. The normal modes of a rotating, elliptical earth'ii nearresonance multiplet coupling, Geophys. J. Int., 18(4), 397-436.

Dahlen, F., 1972. Elastic dislocation theory for a self-gravitating elastic configuration with an initial static stress field, Geophys. J. Int., 28(4), 357-383.

Dahlen, F.A., 1968. The normal modes of a rotating, elliptical earth, Geophys. J. Int., 16(4), 329-367.

Dahlen, F.A., 1976. The passive influence of the oceans upon the rotation of the earth, Geophys. J. Int., 46(2), 363-406.

Dahlen, F.A. \& Tromp, J., 1998. Theoretical Global Seismology, Princeton University Press.

Deuss, A. \& Woodhouse, J., 2004. Iteration method to determine the eigenvalues and eigenvectors of a target multiplet including full mode coupling, Geophys. J. Int., 159(1), 326-332.

Deuss, A. \& Woodhouse, J.H., 2001. Theoretical free-oscillation spectra: the importance of wide band coupling, Geophys. J. Int., 146(3), 833-842.

Dziewonski, A.M. \& Anderson, D.L., 1981. Preliminary reference Earth model, Phys. Earth planet. Inter, 25(4), 297-356.

Ebin, D.G. \& Marsden, J., 1970. Groups of diffeomorphisms and the motion of an incompressible fluid, Ann. Math., 92, 102-163.

Faccioli, E., Maggio, F., Paolucci, R. \& Quarteroni, A., 1997. 2d and 3d elastic wave propagation by a pseudo-spectral domain decomposition method, J. Seismology, 1(3), 237-251.

Farrell, W.E. \& Clark, J.A., 1976. On postglacial sea level, Geophys. J. Int., 46(3), 647-667.

Gharti, H.N. \& Tromp, J., 2017. A spectral-infinite-element solution of poisson's equation: an application to self gravity, preprint arXiv:1706.00855.

Gilbert, F., 1971. Excitation of the normal modes of the earth by earthquake sources, Geophys. J. Int., 22(2), 223-226.

Greenleaf, A., Lassas, M. \& Uhlmann, G., 2003. Maths. Res. Lett., 10, 685-693.

Hara, T., Tsuboi, S. \& Geller, R.J., 1991. Inversion for laterally heterogeneous earth structure using a laterally heterogeneous starting model: preliminary results, Geophys. J. Int., 104(3), 523-540.

Hara, T., Tsuboi, S. \& Geller, R.J., 1993. Inversion for laterally heterogeneous upper mantle s-wave velocity structure using iterative waveform inversion, Geophys. J. Int., 115(3), 667-698.

Holm, D.D., Schmah, T. \& Stoica, C., 2009. Geometric Mechanics and Symmetry: From Finite to Infinite Dimensions, Vol. 12, Oxford University Press.

Holzapfel, G.A., 2000. Nonlinear Solid Mechanics, Wiley.

Holzapfel, G.A., 2002. Nonlinear solid mechanics: a continuum approach for engineering science, Meccanica, 37(4), 489-490.

Ishii, M. \& Tromp, J., 1999. Normal-mode and free-air gravity constraints on lateral variations in velocity and density of earth9s mantle, Science, 285(5431), 1231-1236.

Jeffreys, H., 1976. The Earth. Its Origin, History and Physical Constitution, 6th edn, Cambridge Univ. Press, 12+ 574 pp.

Jobert, G., 1976. Matrix methods for generally stratified media, Geophys. J. Int., 47(2), 351-362.

Komatitsch, D. \& Tromp, J., 1999. Introduction to the spectral element method for three-dimensional seismic wave propagation, Geophys. J. Int., 139(3), 806-822.

Komatitsch, D. \& Tromp, J., 2002. Spectral-element simulations of global seismic wave propagation'ii. three-dimensional models, oceans, rotation and self-gravitation, Geophys. J. Int., 150(1), 303-318.

Komatitsch, D. \& Vilotte, J.-P., 1998. The spectral element method: an efficient tool to simulate the seismic response of $2 \mathrm{~d}$ and $3 \mathrm{~d}$ geological structures, Bull. seism. soc. Am., 88(2), 368-392.

Lau, H.C., Yang, H.-Y., Tromp, J., Mitrovica, J.X., Latychev, K. \& Al-Attar, D., 2015. A normal mode treatment of semi-diurnal body tides on an aspherical, rotating and anelastic earth, Geophys. J. Int., 202(2), 13921406.

Lau, H.C., Mitrovica, J.X., Davis, J.L., Tromp, J., Yang, H.-Y. \& Al-Attar, D., 2017. Tidal tomography constrains earth's deep-mantle buoyancy, Nature, $\mathbf{5 5 1}(7680), 321$.
Lay, T., 2007. Deep earth structure - lower mantle and D”, Romanowicz, B. \& Dziewonski, A. in Treatise on Geophysics, Vol 1: Seismology and Structure of the Earth, Elsevier, pp. 619-654.

Lock, S.J. \& Stewart, S.T., 2017. The structure of terrestrial bodies: impact heating, corotation limits, and synestias, J geophys Res., 122(5), 950-982.

Lognonné, P., 1991. Normal modes and seismograms in an anelastic rotating earth, J. geophys. Res., 96(B12), 20 309-20 319.

Lognonné, P. \& Romanowicz, B., 1990. Modelling of coupled normal modes of the earth: the spectral method, Geophys. J. Int., 102(2), 365-395.

Marsden, J.E. \& Hughes, T.J., 1994. Mathematical Foundations of Elasticity, Courier Corporation.

Mazzucato, A.L. \& Rachele, L.V., 2006. Partial uniqueness and obstruction to uniqueness in inverse problems for anisotropic elastic media, J. Elast., 83(3), 205-245.

Moczo, P., 1989. Finite-difference technique for sh-waves in 2-d media using irregular grids'application to the seismic response problem, Geophys. J. Int., 99(2), 321-329.

Moulik, P. \& Ekström, G., 2016. The relationships between large-scale variations in shear velocity, density, and compressional velocity in the earth's mantle, J. geophys. Res., 121(4), 2737-2771.

Noll, W., 1974. The Foundations of Mechanics and Thermodynamics: Selected Papers, Springer.

Omori, H., 1970. On the group of diffeomorphisms on a compact manifold, Proc. Symp. Pure Math., , in Proc. Symp. Pure Appl. Math., Vol. 15, pp. 167-183, Am. Math. Soc.

Omori, H., 1974. Infinite-Dimensional Lie Groups, American Mathematical Soc.

Park, J., 1990. The subspace projection method for constructing coupledmode synthetic seismograms, Geophys. J. Int., 101(1), 111-123.

Park, J. \& Gilbert, F., 1986. Coupled free oscillations of an aspherical, dissipative, rotating earth: Galerkin theory, J. geophys. Res., 91(B7), 72417260.

Pekeris, C. \& Jarosch, H., 1958. The free oscillations of the earth, in Contributions in Geophysics in Honor of Beno Gutenberg. eds Benioff, H., Ewing, M., Howell, B. \& Press, F., Pergamon Press.

Pendry, J.B., Schurig, D. \& Smith, D.R., 2006. Controlling electromagnetic fields, Science, 312(5781), 1780-1782.

Rahm, M., Schurig, D., Roberts, D.A., Cummer, S.A., Smith, D.R. \& Pendry, J.B., 2008. Design of electromagnetic cloaks and concentrators using form-invariant coordinate transformations of maxwell's equations, Photonics Nanostructures - Fundam. Appl., 6(1), 87-95.

Rogister, Y. \& Valette, B., 2009. Influence of liquid core dynamics on rotational modes, Geophys. J. Int., 176(2), 368-388.

Romanowicz, B., 1987. Multiplet-multiplet coupling due to lateral heterogeneity: asymptotic effects on the amplitude and frequency of the earth's normal modes, Geophys. J. Int., 90(1), 75-100.

Seriani, G., Priolo, E., Pregarz, A. \& Cohen, G., 1995. Modelling waves in anisotropic media by a spectral element method, in Proceedings of the Third International Conference on Mathematical and Numerical Aspects of Wave Propagation, Cohen, G., SIAM, pp. 289-298.

Simpson, H.C. \& Spector, S.J., 1987. On the positivity of the second variation in finite elasticity, Arch. Ration. Mech. Anal., 98(1), 1-30.

Stefanov, P. \& Uhlmann, G., 1998a. Rigidity for metrics with the same lengths of geodesics, Math. Res. Lett., 5, 83-96.

Stefanov, P. \& Uhlmann, G., 1998b. Stability estimates for the hyperbolic dirichlet to neumann map in anisotropic media, J. Funct. Anal., 154(2), 330-358.

Takeuchi, N., 2005. Finite boundary perturbation theory for the elastic equation of motion, Geophys. J. Int., 160(3), 1044-1058.

Thompson, J.L., 1969. Some existence theorems for the traction boundary value problem of linearized elastostatics, Arch. Ration. Mechan. Anal., 32(5), 369-399.

Trampert, J., Deschamps, F., Resovsky, J. \& Yuen, D., 2004. Probabilistic tomography maps chemical heterogeneities throughout the lower mantle, Science, 306(5697), 853-856.

Tromp, J. \& Dahlen, F., 1990. Summation of the born series for the normal modes of the earth, Geophys. J. Int., 100(3), 527-533. 
Truesdell, C. \& Noll, W., 2004., The Non-Linear Field Theories of Mechanics, pp. 1-579, Springer.

Um, J. \& Dahlen, F., 1992. Normal mode multiplet coupling on an aspherical, anelastic earth, Geophys. J. Int., 111(1), 11-31.

Valentine, A.P. \& Trampert, J., 2015. The impact of approximations and arbitrary choices on geophysical images, Geophys. J. Int., 204(1), 59-73.

Valette, B., 1989a. Etude d'une classe de problèmes spectraux, C.R. Acad. Sci., Paris, 309(12), 785-788.

Valette, B., 1989b. Spectre des vibrations propres d'un corps élastique, autogravitant, en rotation uniforme et contenant une partie fluide, C.R. Acad. Sci., Paris, 309, 419-422.

Virieux, J., 1986. P-sv wave propagation in heterogeneous media: Velocitystress finite-difference method, Geophysics, 51(4), 889-901.

Woodhouse, J., 1976. On rayleigh's principle, Geophys. J. Int., 46(1), 11-22.

Woodhouse, J., 1980. The coupling and attenuation of nearly resonant multiplets in the earth's free oscillation spectrum, Geophys. J. Int., 61(2), 261-283.
Woodhouse, J., 1983. The joint inversion of seismic waveforms for lateral variations in earth structure and earthquake source parameters, in Proc. Enrico Fermi Int. Sch. Phys, Vol. 85, pp. 366-397, Kanamori, H. \& Boschi, E., Societa Italiana di Fisica.

Woodhouse, J., 1988. The calculation of eigenfrequencies and eigenfunctions of the free oscillations of the earth and the sun, in Seismological Algorithms, pp. 321-370, Doornbos, D.J., Academic Press.

Woodhouse, J. \& Dahlen, F., 1978. The effect of a general aspherical perturbation on the free oscillations of the earth, Geophys. J. Int., 53(2), 335-354.

Woodhouse, J. \& Girnius, T., 1982. Surface waves and free oscillations in a regionalized earth model, Geophys. J. Int., 68(3), 653-673.

Woodhouse, J.H. \& Deuss, A., 2007. Earth's free oscillations, in Treatise on Geophysics, Seismology and Structure of the Earth, Vol. 1, pp. 31-65, eds Romanowicz, B. \& Dziewonski, A., Elsevier.

Yang, H.-Y. \& Tromp, J., 2015. Synthetic free-oscillation spectra: an appraisal of various mode-coupling methods, Geophys. J. Int., 203(2), 11791192. 